\title{
FNDC5 alleviates oxidative stress and cardiomyocyte apoptosis in doxorubicin-induced cardiotoxicity via activating AKT
}

\author{
Xin Zhang ${ }^{1,2,3} \cdot$ Can $\mathrm{Hu}^{1,2,3} \cdot$ Chun-Yan Kong ${ }^{1,2,3} \cdot$ Peng Song ${ }^{1,2,3} \cdot$ Hai-Ming $\mathrm{Wu}^{1,2,3} \cdot$ Si-Chi $\mathrm{Xu}^{1,2,3} \cdot$ Yu-Pei Yuan ${ }^{1,2,3}$. \\ Wei Deng ${ }^{1,2,3} \cdot$ Zhen-Guo Ma $^{1,2,3} \cdot$ Qi-Zhu Tang ${ }^{1,2,3}$
}

Received: 17 December 2018 / Revised: 11 May 2019 / Accepted: 3 June 2019 / Published online: 17 June 2019

(c) The Author(s) 2019. This article is published with open access

\begin{abstract}
Oxidative stress and cardiomyocyte apoptosis play critical roles in doxorubicin (DOX)-induced cardiotoxicity. Previous studies indicated that fibronectin type III domain-containing 5 (FNDC5) and its cleaved form, irisin, could preserve mitochondrial function and attenuate oxidative damage as well as cell apoptosis, however, its role in DOX-induced cardiotoxicity remains unknown. Our present study aimed to investigate the role and underlying mechanism of FNDC5 on oxidative stress and cardiomyocyte apoptosis in DOX-induced cardiotoxicity. Cardiomyocyte-specific FNDC5 overexpression was achieved using an adeno-associated virus system, and then the mice were exposed to a single intraperitoneal injection of DOX $(15 \mathrm{mg} / \mathrm{kg})$ to generate DOX-induced cardiotoxicity. Herein, we found that FNDC5 expression was downregulated in DOX-treated murine hearts and cardiomyocytes. Fndc5 deficiency resulted in increased oxidative damage and apoptosis in $\mathrm{H} 9 \mathrm{C} 2$ cells under basal conditions, imitating the phenotype of DOX-induced cardiomyopathy in vitro, conversely, FNDC5 overexpression or irisin treatment alleviated DOX-induced oxidative stress and cardiomyocyte apoptosis in vivo and in vitro. Mechanistically, we identified that FNDC5/Irisin activated AKT/mTOR signaling and decreased DOX-induced cardiomyocyte apoptosis, and moreover, we provided direct evidence that the anti-oxidant effect of FNDC5/Irisin was mediated by the AKT/GSK3 $\beta / F Y N / N r f 2$ axis in an mTOR-independent manner. And we also demonstrated that heat shock protein 20 was responsible for the activation of AKT caused by FNDC5/Irisin. In line with the data in acute model, we also found that FNDC5/Irisin exerted beneficial effects in chronic model of DOX-induced cardiotoxicity ( $5 \mathrm{mg} / \mathrm{kg}$, i.p., once a week for three times, the total cumulative dose is $15 \mathrm{mg} / \mathrm{kg}$ ) in mice. Based on these findings, we supposed that FNDC5/Irisin was a potential therapeutic agent against DOX-induced cardiotoxicity.
\end{abstract}

These authors contributed equally: Xin Zhang, Can Hu

Edited by S. Kaufmann

Supplementary information The online version of this article (https:// doi.org/10.1038/s41418-019-0372-z) contains supplementary material, which is available to authorized users.

Zhen-Guo Ma

zhengma@whu.edu.cn

$\bowtie$ Qi-Zhu Tang

qztang@whu.edu.cn

1 Department of Cardiology, Renmin Hospital of Wuhan University, 430060 Wuhan, PR China

2 Cardiovascular Research Institute of Wuhan University, 430060 Wuhan, PR China

3 Hubei Key Laboratory of Cardiology, 430060 Wuhan, PR China

\section{Introduction}

Despite its effectiveness in the treatment of various human cancers, the chemotherapeutic application of doxorubicin (DOX) is largely limited for the cause of life-threatening cardiotoxicity [1, 2]. Multiple factors have been substantiated to be involved in the pathogenesis of DOXinduced cardiotoxicity, including DNA/RNA/protein synthesis inhibition, autophagy dysregulation and the disturbance of intracellular calcium homeostasis, however, emerging studies proposed indispensable roles of reactive oxygen species (ROS) overproduction and cardiomyocyte apoptosis in DOX-induced myocardial damage [3-5]. Excessive ROS induces oxidative damage to biological macromolecules, including lipids, proteins and DNA, and disrupts cellular membrane integrity and function [6]. Moreover, DOX-evoked oxidative stress can directly elicit massive cardiomyocyte apoptosis via both extrinsic and 
intrinsic apoptotic pathways and cause severe cardiac dysfunction [7, 8]. Previous studies also demonstrated that DOX could induce apoptosis via mechanisms that do not directly involve ROS production and oxidative stress [8]. Our recent study also revealed that suppression of cardiomyocyte apoptosis significantly attenuated DOX-induced cardiac dysfunction [9]. Therefore, an intensive understanding of the pathogenesis of DOX-induced oxidative stress and cardiomyocyte apoptosis, and the identification of novel therapeutic targets are urgently needed.

Protein kinase B (PKB/AKT) is a serine/threonine protein kinase involved in the regulation of cell survival, proliferation, and metabolism and is inhibited in DOX-treated murine hearts [10]. Increasing studies proved that activation of AKT prevented cardiomyocyte apoptosis in response to DOX, whereas AKT inhibition exaggerated DOX-induced cardiomyocyte apoptosis and cardiac dysfunction [11, 12]. The mammalian target of rapamycin (mTOR) is a classical and important downstream mediator in AKT signaling pathway, which exerts beneficial role in regulating protein synthesis and cell survival via P70 S6 kinase (P70) and 4Ebinding protein 1 (4EBP1) [13]. Zhu et al. found that mTOR inhibition was the major contributor to DOXtriggered myocardial mass loss and cardiac dysfunction, and overexpression of constitutively active mTOR could protect DOX-induced cardiotoxicity [14]. In addition to the role in regulating cell survival, current available data suggested that AKT also played a critical role in relieving oxidative stress via deactivating glycogen synthase kinase $3 \beta$ (GSK3 $\beta$ ), which thereby decreases FYN nuclear translocation-mediated NF-E2-related factor 2 (Nrf2) nuclear export and degradation [15-17]. Considering the beneficial role in DOX-induced cardiotoxicity, it is important to unearth a novel positive regulator of AKT.

Fibronectin type III domain-containing protein 5 (FNDC5, also known as FRCP2 and PeP) is a glycosylated transmembrane protein, with a signal peptide, two fibronectin domains and one hydrophobic domain inserted into the cell membrane, that can be cleaved and released as irisin [18]. FNDC5/Irisin was traditionally found in muscle and regarded as a myokine that drives brown-fatlike development of white fat and thermogenesis, which was suggested as a therapeutic agent for human metabolic diseases [19]. Liu et al. found that Fndc5 deficiency aggravated whereas FNDC5 overexpression prevented obesity-related hyperlipemia, hepatic lipid accumulation, and impaired fatty acid oxidation and autophagy in the liver [20]. Except for the beneficial role in metabolic disorders, recent studies also implicated that FNDC5/Irisin was involved in regulating various cardiovascular diseases, such as atherosclerosis, hypertension, myocardial ischemia/reperfusion injury, and cardiac hypertrophy [21-24]. Besides, numerous researches verified that FNDC5 overexpression or irisin supplementation could preserve mitochondrial function and attenuate oxidative damage as well as cell apoptosis [25, 26]. Based on these findings, we hypothesized that FNDC5/Irisin may be a promising candidate for the treatment of DOXinduced cardiotoxicity.

\section{Methods and materials}

\section{Antibodies and reagents}

Antibodies against the following proteins were purchased from Cell Signaling Technology (Danvers, MA, USA): BAX (1:1000), cleaved-Caspase3 (C-Caspase, 1:1000), total Caspase3 (T-Caspase3, 1:1000), total AKT (T-AKT, 1:1000), phosphorylated AKT (P-AKT, 1:1000), T-mTOR (1:1000), P-mTOR (1:1000), T-P70 (1:1000), P-P70 (1:1000), T-ribosomal protein S6 (T-S6, 1:1000), P-S6 (1:1000), T-4EBP1 (1:1000), P-4EBP1 (1:1000), Tglycogen synthase kinase $3 \beta$ (T-GSK3 $\beta, 1: 1000)$, PGSK3 $\beta$ (1:1000), 4-Hydroxynonenal (4-HNE, 1:200 for staining), and glyceraldehyde 3-phosphate dehydrogenase (GAPDH, 1:1000). Antibodies for FNDC5 (1:1000 for western blot, 1:100 for staining), p67phox (1:1000), superoxide dismutase 1 (SOD1, 1:1000), SOD2 (1:1000), B-cell lymphoma 2 (BCL-2, 1:1000), Nrf2 (1:1000), heme oxygenase-1 (HO-1, 1:1000), Kelch-like ECH-associated protein 1 (Keap1, 1:1000), and heat shock protein 20 (HSP20, 1:1000) were purchased from Abcam (Cambridge, UK). Anti-T-FYN (1:200), anti-P-FYN (1:200), and anti-Tproliferating cell nuclear antigen (PCNA, 1:200) were obtained from Santa Cruz Biotechnology (Dallas, TX, USA). The secondary antibody used for western blot was purchased from LI-COR Biosciences, whereas anti-rabbit/ mouse EnVision ${ }^{\mathrm{TM}+} / \mathrm{HRP}$ reagent used for immunohistochemistry was obtained from Gene Technology (Shanghai, China). DOX, irisin, AKT inhibitor (AKT i), rapamycin (Rapa) and dexrazoxane (DEX) were purchased from Sigma-Aldrich (St. Louis, MO, USA). Dihydroethidium (DHE) was obtained from Keygen Biotech, and 2',7'dichlorodihydrofluorescein diacetate (DCFH-DA), malondialdehyde (MDA) assay kit, glutathione (GSH) assay kit, total SOD assay kit and NADPH oxidase assay kit were all purchased from Nanjing Jiancheng Bioengineering Institute (Nanjing, China). Phosphoinositide 3-kinase (PI3K) activity ELISA assay kit was obtained from Echelon Biosciences Inc. ApopTag ${ }^{\circledR}$ Plus In Situ Apoptosis Fluorescein Detection Kit was purchased from Millipore (Billerica, MA, USA) and the cell counting kit-8 (CCK-8) was obtained from Dōjindo Laboratories (Kumamoto, Japan). 


\section{Animals and treatments}

All animal care and experimental procedures were in compliance with the Guidelines for the Care and Use of Laboratory Animals published by the United States National Institutes of Health (NIH Publication, revised 2011) and approved by the Animal Care and Use Committee of Renmin Hospital of Wuhan University. C57BL/6 male mice (8-10 weeks old, 23.5-27.5 g) were purchased from the Institute of Laboratory Animal Science, Chinese Academy of Medical Sciences (Beijing, China) and were subjected to an adaptive feeding for 1 week before the study commenced. All mice were maintained under specific pathogen-free, environmentally controlled (Temperature: $20-25^{\circ} \mathrm{C}$; Humidity: $50 \pm 5 \%$ ) barrier conditions in individual ventilated cages and were fed with sterile food and water ad libitum. To specifically overexpress FNDC5 in the myocardium, mice received a single intravenous injection of adeno-associated virus 9 (AAV9) carrying human FNDC5 under the cTnT promoter (AAV9-FNDC5) or a negative control (AAV9-NC) via the tail vein at a concentration of $1 \times 10^{11}$ viral genome per mouse [9]. The AAV9-FNDC5 and AAV9-NC were generated by Hanbio Biotechnology Co. (Shanghai, China). Four weeks post-AAV9 injection, the mice were exposed to a single intraperitoneal injection of DOX $(15 \mathrm{mg} / \mathrm{kg})$ to generate DOX-induced cardiotoxicity or equal volume of normal saline (NS) as a control referring to our previous study [9]. These mice were observed daily and weighed every 2 days, and then were sacrificed with an overdose of sodium pentobarbital $(200 \mathrm{mg} / \mathrm{kg}$; i.p.) after 8day DOX insult. Murine hearts together with the tibia were collected to calculate the heart weight/tibia length ratios (HW/TL). To verify the hypothesis that the beneficial roles of FNDC5 were dependent on the activation of AKT, mice were intraperitoneally injected with AKT i $(20 \mathrm{mg} / \mathrm{kg} /$ day $)$ for consecutive 14 days from the 6th day before DOX insult [27]. For mTOR activity inhibition, mice were daily treated with rapamycin $(5 \mathrm{mg} / \mathrm{kg} / \mathrm{day})$ for 14 days as AKT i via intraperitoneal injection according to previous studies $[20,28]$. To verify the role of Nrf2 and HSP20 in vivo, mice were exposed to a single intravenous injection of AAV9 carrying small hairpin RNA against Nrf2 (sh Nrf2) or HSP20 (sh Hsp2O) or their corresponding negative control (sh RNA) 2 weeks before FNDC5 overexpression. To investigate the therapeutic effect of irisin, mice were subcutaneously infused with either saline or irisin $(12 \mathrm{nmol} / \mathrm{kg} /$ day) for 14 days from the 6th day before DOX injection via osmotic minipumps (Alzet model 2004, Alza Corp) as previously described $[29,30]$. In addition, mice were intraperitoneally injected with a weekly dose of $60 \mathrm{mg} / \mathrm{kg}$ DEX for two times, with the first injection at the 6th day before DOX injection, and the second one at the second day after DOX treatment [31].
To enhance the clinical impact of our current work, mice were injected intraperitoneally with DOX $(5 \mathrm{mg} / \mathrm{kg}$, once a week, the total cumulative dose is $15 \mathrm{mg} / \mathrm{kg}$ ) for three times to generate chronic model of DOX-induced cardiotoxicity [32]. After 6 weeks from the first injection of DOX, cardiac function was evaluated and murine hearts were collected for further detection. Four weeks before DOX injection, mice received a single intravenous injection of AAV9-FNDC5 to overexpress FNDC5 in myocardium or AAV9-NC as a control. All these mice were observed daily and the survival rate was calculated every week. To compare the effect of irisin and DEX in chronic model, mice with irisin protection were pretreated with irisin $(12 \mathrm{nmol} / \mathrm{kg} /$ day $)$ for 6 days and lasting for another 6 weeks, whereas mice with DEX administration were intraperitoneally injected with a weekly dose of $60 \mathrm{mg} / \mathrm{kg}$ DEX for seven times [29-31]. Considering the fact that long-term use of high-doses of DEX resulted in severe side effects, we then investigated whether combined use of DEX with irisin $(6 \mathrm{nmol} / \mathrm{kg} /$ day $)$ could decrease the usage of DEX. In this experiment, mice were exposed to DEX (60 mg/kg/week), DEX (30 mg/kg/week), or DEX + irisin $(30 \mathrm{mg} / \mathrm{kg} /$ week for DEX, $6 \mathrm{nmol} / \mathrm{kg} / \mathrm{day}$ for irisin) in the presence of chronic DOX insult.

\section{Echocardiography and hemodynamics}

Transthoracic echocardiography was performed according to our previous studies, and echocardiographic parameters were averaged from three to five cardiac cycles [33-37]. Particular attention was given not to bring excessive pressure to the chest, which could cause bradycardia and deformation of the heart. Invasive hemodynamic monitoring was performed by PowerLab system (AD Instruments Ltd., Oxford, UK) using a 1.4-French Millar pressure-volume catheter (SPR-839; Millar Instruments, Houston, TX).

\section{Western blot and quantitative real-time PCR}

Western blot and quantitative real-time PCR were performed referring to our previous articles [33-37]. Nuclear protein fractions were separated by a commercial kit as our previously described and were normalized to PCNA $[9,33]$. Total RNA was extracted using TRIzol reagent and reverse transcribed with Maxima First Strand cDNA Synthesis Kit [Roche (Basel, Switzerland), 04896866001]. The expression level of each individual transcript was normalized to Gapdh.

\section{Immunohistochemistry and TdT-mediated dUTP nick end-labeling (TUNEL) staining}

Immunohistochemistry staining was performed according to our previous studies $[38,39]$. Endogenous peroxidase and 
the nonspecific binding of the antibody were blocked with $3 \%$ hydrogen peroxide or $10 \%$ goat serum, respectively. Sections were determined by the light microscopy (Nikon (Tokyo, Japan), H550L) in a blinded manner. TUNEL staining was performed to detect cell apoptosis both in vivo and in vitro according to the manufacturer's instructions using a commercially available kit, and the images were captured by a special OLYMPUS DX51 fluorescence microscope (Tokyo, Japan).

\section{Cell culture and treatments}

H9C2 cells were purchased from the Cell Bank of the Chinese Academy of Sciences (Shanghai, China) and were cultured in Dulbecco's modified Eagle's medium with $10 \%$ fetal bovine serum. After synchronization for $24 \mathrm{~h}$, cells were treated with irisin $(20 \mathrm{nmol} / \mathrm{L})$ in the presence or absence of DOX $(1 \mu \mathrm{mol} / \mathrm{L})$ for $24 \mathrm{~h}[9,24]$. For AKT or mTOR inhibition, $\mathrm{H} 9 \mathrm{C} 2$ cells were pretreated with AKT i $(1 \mu \mathrm{mol} / \mathrm{L})$, or rapamycin $(50 \mathrm{nmol} / \mathrm{L})$ for $30 \mathrm{~min}$ [20, 24, 38]. To knockdown the expression of FNDC5 or $\mathrm{Nrf2}$, cells were transfected with scramble small interfering RNA (si $R N A, 50 \mathrm{nmol} / \mathrm{L})$, si $F n d c 5(50 \mathrm{nmol} / \mathrm{L})$ or si $N r f 2(50 \mathrm{nmol} / \mathrm{L})$ for $4 \mathrm{~h}$ using Lipo6000 ${ }^{\mathrm{TM}}$ transfection reagent according to the manufacturer's protocol (RiboBio Co. Ltd, Guangzhou, Guangdong, China), and then were cultured in normal medium for $24 \mathrm{~h}$ before the next treatment $[9,33]$.

\section{Oxidative stress detection and cell viability}

ROS production was evaluated by DHE staining in vivo and DCFH-DA staining in vitro [38, 40]. Briefly, cryosections of fresh heart samples or coverslips were stained with DHE $(5 \mu \mathrm{mol} / \mathrm{L})$ or DCFH-DA $(5 \mu \mathrm{mol} / \mathrm{L})$ in the dark at $37^{\circ} \mathrm{C}$ for $30 \mathrm{~min}$, and then were visualized in a blinded manner under an Olympus IX53fluorescence microscope. To further assess oxidative stress level, we measured the content of MDA, GSH, total SOD activity and NADPH oxidase activity in the myocardium or $\mathrm{H} 9 \mathrm{C} 2$ cells according to our previous study by the commercially available kits [38]. Cell viability was determined using the CCK- 8 assay kit according to the manufacturer's protocol as described previously $[9,38]$.

\section{Biochemical determination}

Serum was collected and the concentrations of cardiac isoform of Tropnin $\mathrm{T}$ (cTnT), lactate dehydrogenase (LDH) and creatine kinase isoenzymes (CK-MB) were measured by an automatic biochemical analyzer (ADVIA ${ }^{\circledR} 2400$, Siemens Ltd., China) [41].

\section{Statistical analysis}

All data in this research were expressed as mean \pm standard error of the mean (SEM) and analyzed by SPSS22.0 software. One way analysis of variance (ANOVA) followed by Tukey post hoc test was performed when comparing multiple groups, whereas differences in two groups were evaluated using unpaired Student's $t$-test. Survival data were assessed by the Kaplan-Meier method and survival curves were compared using the Mantel-Cox log-rank test. A $P$ value $<0.05$ was considered statistically significant.

\section{Results}

\section{FNDC5 attenuated DOX-induced cardiac dysfunction in mice}

Previous studies reported that FNDC5 was mainly expressed in skeletal muscle [18], however, we found that the relative mRNA expression of $F n d c 5$ was more abundant in the myocardium than that in skeletal muscle, which was downregulated in response to DOX administration (Fig. S1a-b). Immunohistochemistry staining showed that FNDC5 in the heart was mainly localized to cardiomyocytes, and the protein level was also significantly decreased in DOX-treated hearts, which was further confirmed by the western blot results (Fig. S1c-d). In addition, DOX incubation suppressed FNDC5 expression in isolated cardiomyocytes (Fig. S1e).

To explore the role of FNDC5 in DOX-induced cardiotoxicity, we specifically overexpressed FNDC5 in myocardium via a single injection of an AAV9 carrying FNDC5 under the cTnT promoter. As shown in Fig. S1f, the myocardial mRNA level of FNDC5 was markedly increased in mice infected with AAV9-FNDC5. Besides, myocardial FNDC5 protein level was restored to normal level in the presence of DOX, which is moderate and efficacious, therefore we selected this time for the next experiments (Fig. S1g). As shown in Fig. 1a, b, FNDC5 overexpression prevented DOX-induced cardiac dysfunction, as indicated by the preserved fractional shortening (FS) and $\pm \mathrm{d} p / \mathrm{d} t$. Besides, we also observed that DOX injection decreased the ratio of heart weight to tibia length (HW/TL), which were significantly attenuated by FNDC5 overexpression (Fig. 1c). Body weight loss due to chemotherapeutic agents and cachexia syndrome predicts bad prognosis in cancer patients via compromising functions of skeletal muscle, adipose tissue and also internal organs, including the liver, kidneys, lungs, especially the heart [42]. Previous studies indicated that DOX application significantly decreased the body weight in cancer patients [43], but intriguingly, we 
Fig. 1 FNDC5 attenuated doxorubicin (DOX)-induced cardiac dysfunction in mice. a Fractional shortening (FS) of mice as determined via echocardiography 8 days after DOX injection $(n=8)$. b Hemodynamic parameter of mice with or without FNDC5 overexpression $(n=8)$. c Statistical results of the heart weight/tibia length ((HW/TL) $(n=8)$. (d) Body weight alterations $(n=8)$. $\mathbf{e}-\mathbf{g}$ Biochemical determination of cTnT, LDH, CK-MB serum levels $(n=10)$. Values represent the mean \pm SEM. $* P<0.05$ versus the corresponding normal saline (NS) group mice injected with negative control (NC) adeno-associated virus $9,{ }^{\#} P<$ 0.05 versus DOX-treated mice injected with AAV9-NC a

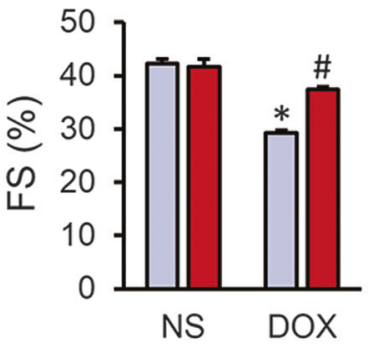

C
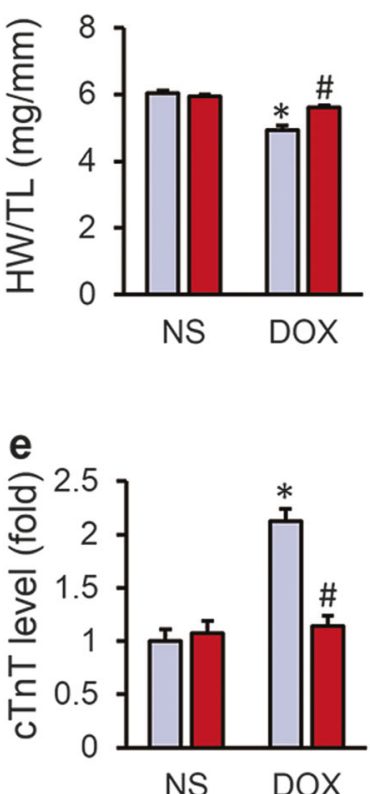

b

AAV9-NC

AAV9-FNDC5
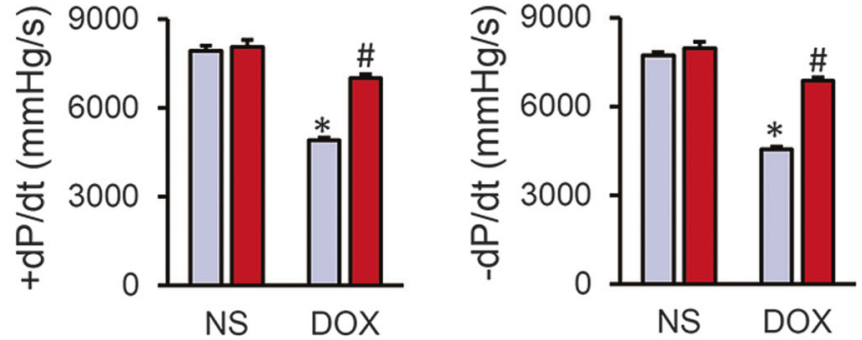

d

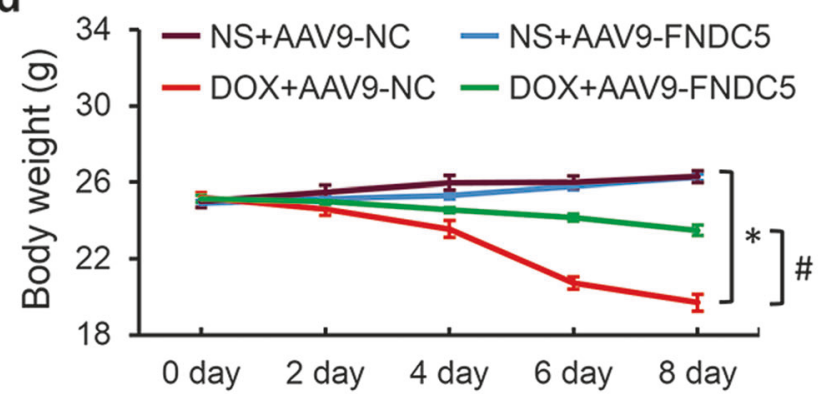

f
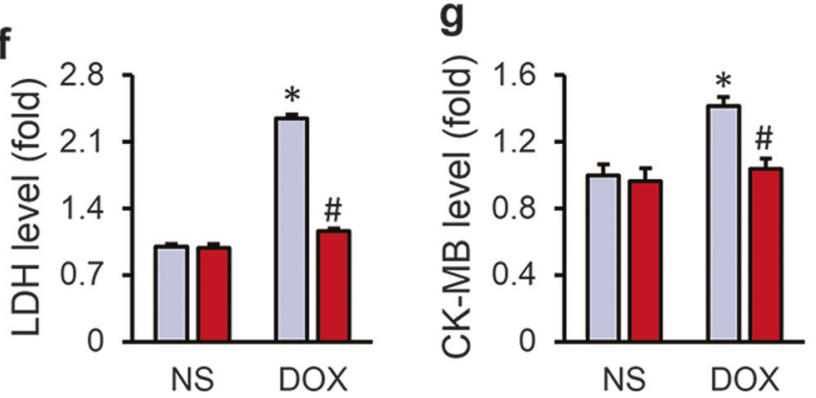

found that cardiac-specific overexpression of FNDC5 attenuated DOX-induced body weight loss in mice, which raises the possibility for its clinical use (Fig. 1d). Myocardial injury was also assessed by the serum levels of cTnT, LDH, and CK-MB, and we observed that FNDC5 could alleviate DOX-induced myocardial injury (Fig. 1e-g). Collectively, we concluded that FNDC5 overexpression attenuated DOX-induced cardiotoxicity in mice.

\section{FNDC5 protected heart from oxidative damage in response to DOX insult}

Oxidative damage is suggested to be the main cause of DOX-induced cardiotoxicity, thus we detected oxidative stress level in the heart via DHE and 4-HNE staining. DOX injection resulted in increased oxidative stress in the heart, and FNDC5 overexpression almost completely inhibited ROS production (Fig. 2a, b). In addition, western blot showed that FNDC5 overexpression significantly decreased the NADPH oxidase subunit p67phox and increased SOD1 and SOD2 expression in DOX-treated hearts (Fig. 2c, d). Consistent with the molecular alterations, we found that FNDC5 overexpression reduced the abnormal MDA level and NADPH oxidase activity, and preserved GSH level and total SOD activity (Fig. 2e).

\section{FNDC5 suppressed cardiomyocyte apoptosis in DOX- treated mice}

Excessive ROS production and DOX itself could both induce cardiomyocyte apoptosis and contributed to the progression of cardiac dysfunction, and we then assessed the role of FNDC5 in cardiomyocyte apoptosis. As shown in Fig. 2f, g, DOX injection resulted in distinct cardiomyocyte apoptosis in vivo, and overexpression of FNDC5 mitigated this pathological alteration. The inhibitory effects of FNDC5 on cardiomyocyte apoptosis were further confirmed by western blot results showing that FNDC5 decreased the expression of BAX, C-Caspase 3 and increased the BCL-2 level (Fig. 2h, i). 
a

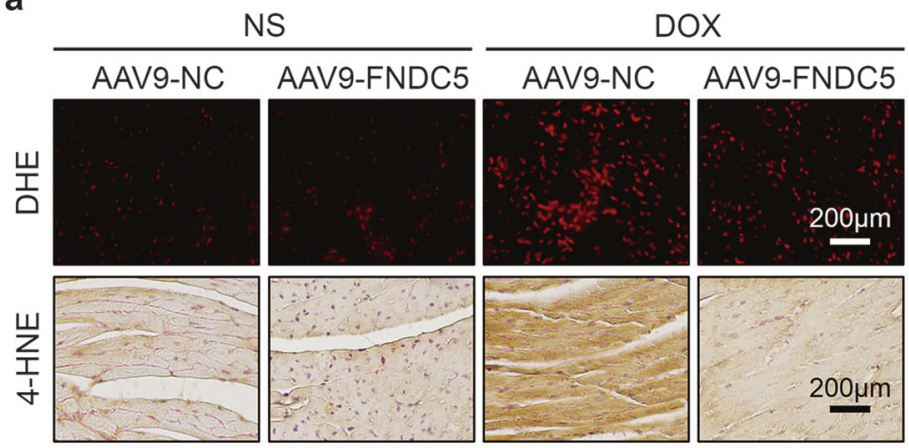

b
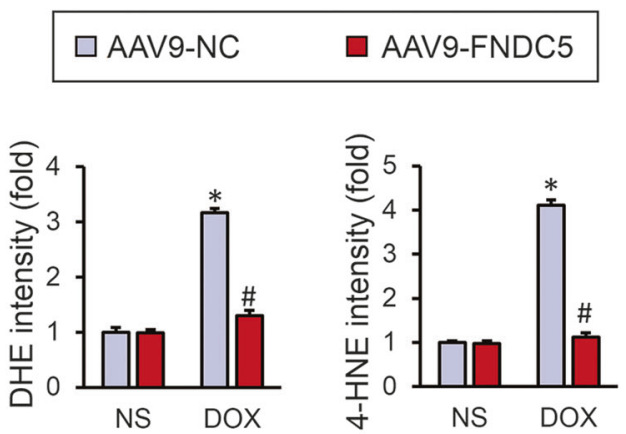

C

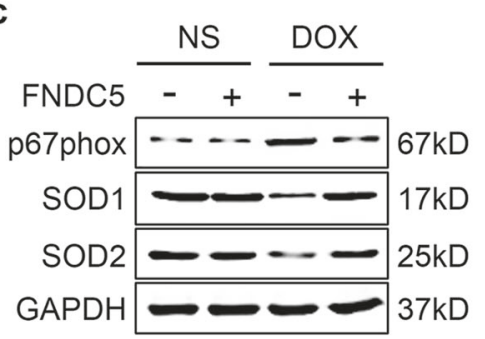

d
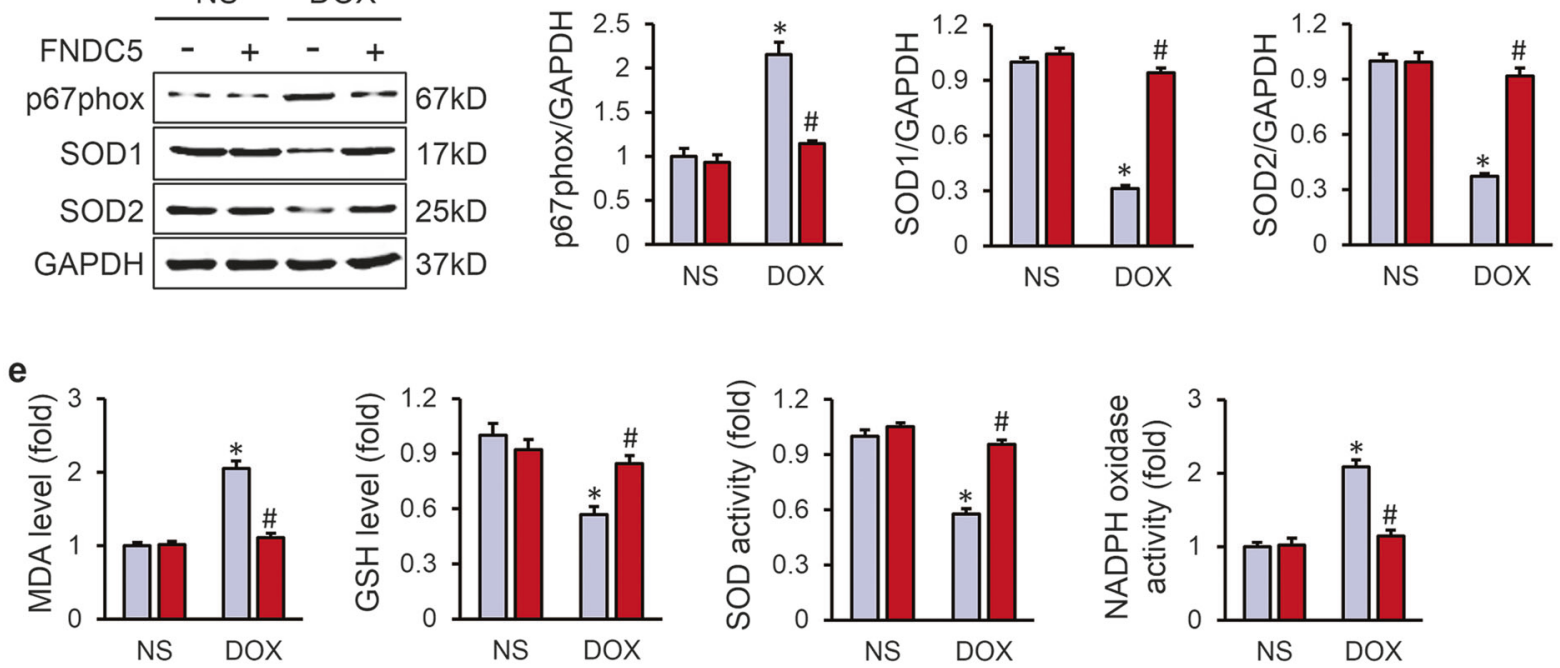

f

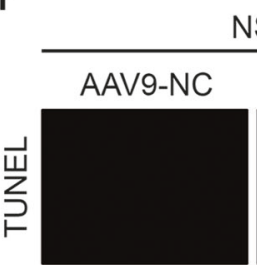

NS

DOX
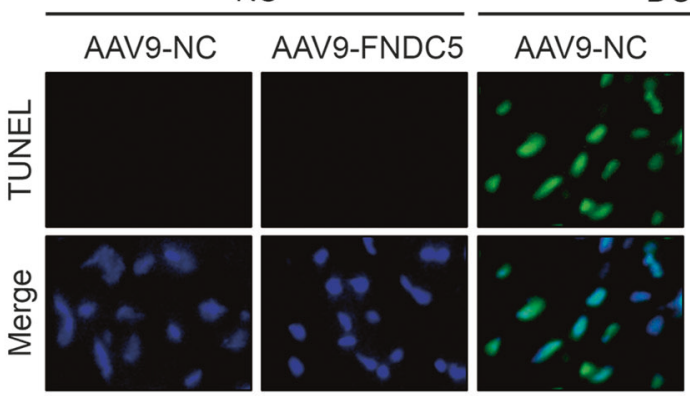

AAV9-FNDC5

h
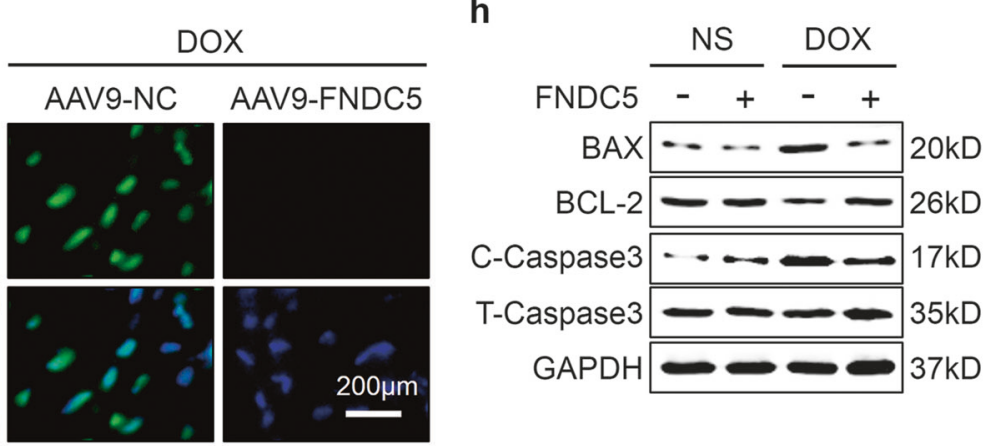
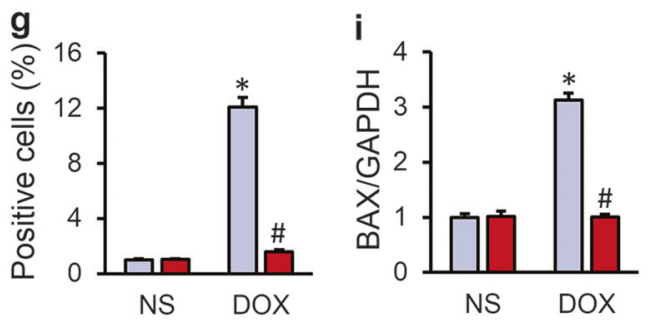

Fig. 2 FNDC5 suppressed oxidative stress and cardiomyocyte apoptosis in DOX-treated mice. a, b Representative DHE, 4-HNE staining images and the quantitative results $(n=6)$. c, d Western blots and statistical results $(n=6)$. e Quantitative results of myocardial MDA, GSH levels and SOD, NADPH oxidase activities $(n=6)$. f, $\mathbf{g}$ TUNEL
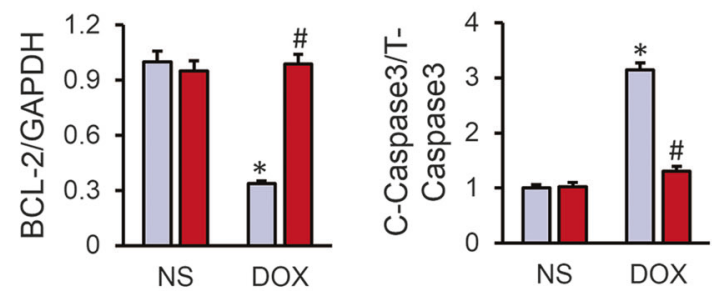

staining and the quantitative results $(n=8)$. $\mathbf{h}, \mathbf{i}$ Western blots and the statistical results of apoptosis-related proteins $(n=6)$. Values represent the mean \pm SEM. $* P<0.05$ versus the corresponding normal saline (NS) group mice injected with negative control (NC) adeno-associated virus $9,{ }^{\#} P<0.05$ versus DOX-treated mice injected with AAV9-NC 
a

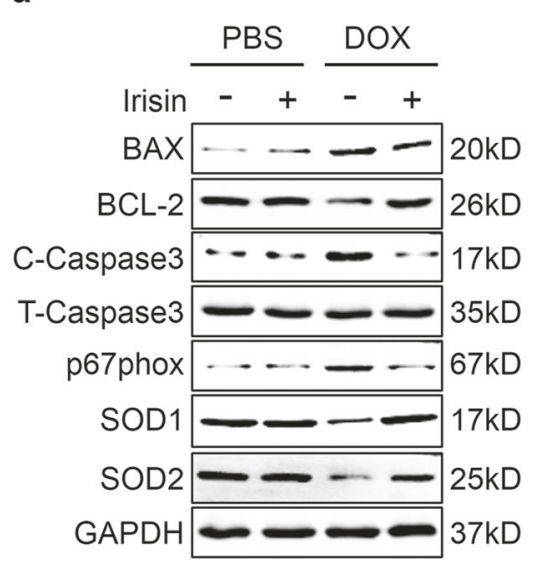

b
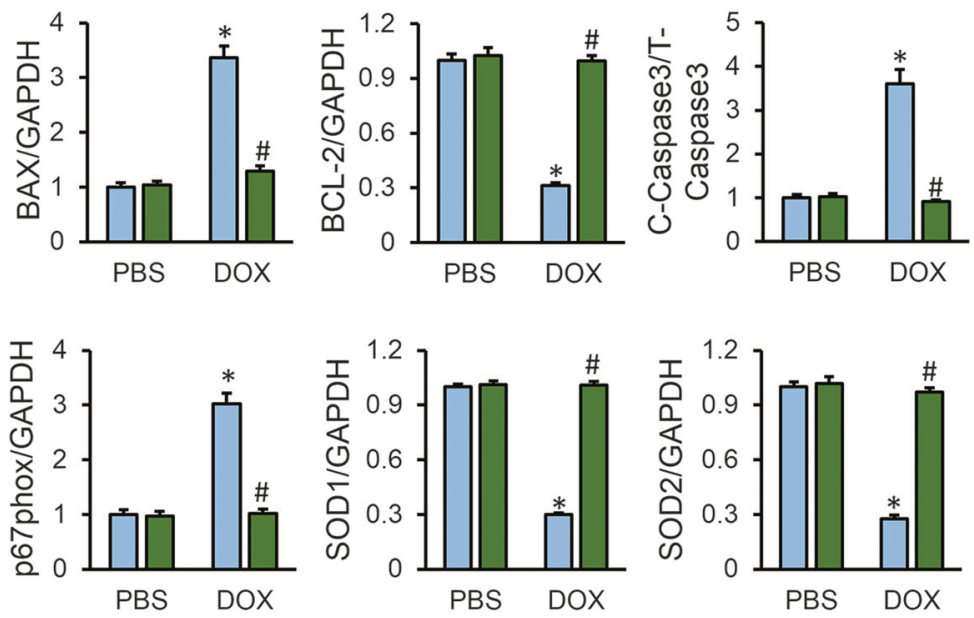

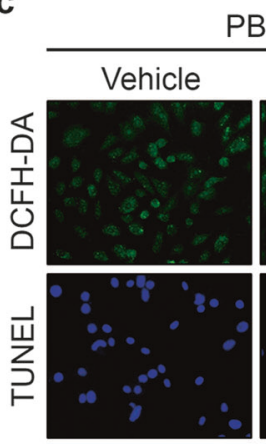

PBS

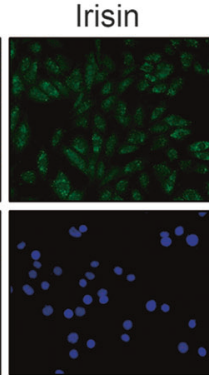

DOX

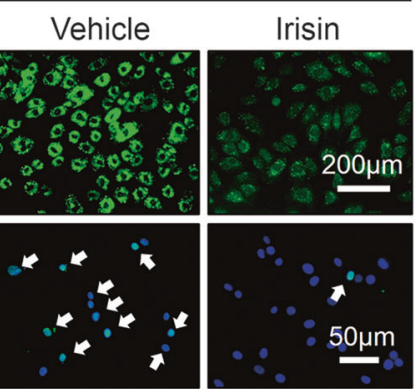

d

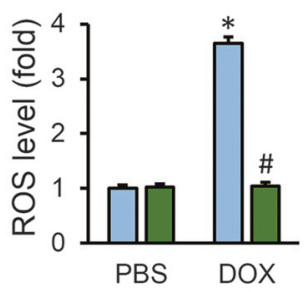

f

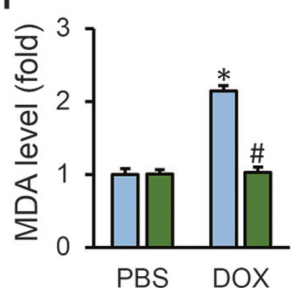

PBS DOX

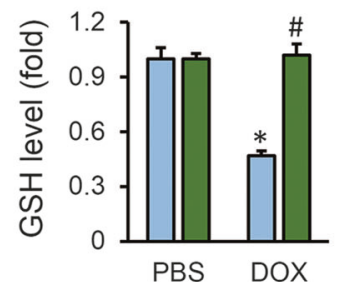

PBS DOX
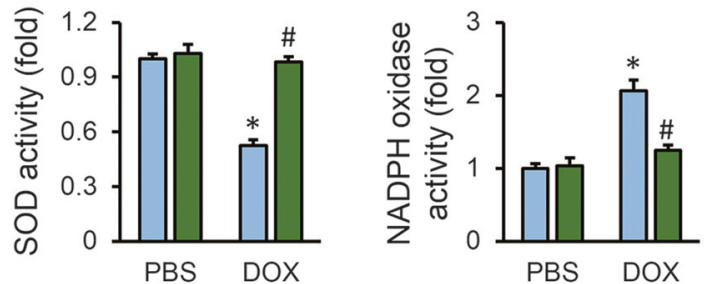

Fig. 3 FNDC5 alleviated DOX-induced oxidative stress and cardiomyocyte apoptosis in vitro. a, b Western blots and statistical results $(n=6)$. c, $\mathbf{d}$ DCFH-DA staining, TUNEL staining and the quantitative results of ROS level $(n=6)$. e Cell viability detected by CCK-8 assay

\section{FNDC5 alleviated DOX-induced oxidative stress and cardiomyocyte apoptosis in vitro}

To further verify the beneficial role of FNDC5 on DOXinduced oxidative stress and cardiomyocyte apoptosis, we treated $\mathrm{H} 9 \mathrm{C} 2$ cells with irisin, a cleaved and secreted fragment of FNDC5, in the presence or absence of DOX in vitro. In line with the data in vivo, we found that irisin showed no effect at baseline, but significantly attenuated DOX-induced upregulation of BAX, C-Caspase3, p67phox and the downregulation of BCL-2, SOD1, and SOD2, and exerted protective effects on oxidative stress and apoptosis in vitro (Fig. 3a,

$(n=6)$. f Quantitative results of MDA, GSH levels and SOD, NADPH oxidase activities in cultured $\mathrm{H} 9 \mathrm{C} 2$ cells $(n=6)$. Values represent the mean \pm SEM. $* P<0.05$ versus PBS group treated with control vehicle, ${ }^{\#} P<0.05$ versus DOX-treated H9C2 cells with irisin protection b). DCFH-DA staining and biochemical analysis further confirmed that irisin treatment alleviated DOX-induced oxidative damage to cardiomyocytes (Fig. 3c-f). TUNEL staining as well as CCK-8 assay results indicated that DOX incubation caused massive apoptosis in $\mathrm{H} 9 \mathrm{C} 2$ cells, which was notably reversed by irisin protection (Fig. 3c, e). Next, we investigated whether Fndc5 deficiency affected oxidative stress and cardiomyocyte apoptosis in vitro. We knocked down the expression of FNDC5 in $\mathrm{H} 9 \mathrm{C} 2$ cells and the efficiency was confirmed by western blot (Fig. S2a, f). Downregulation of FNDC5 resulted in increased cardiomyocyte apoptosis at baseline, as evidenced by the decrease of BCL-2 
a

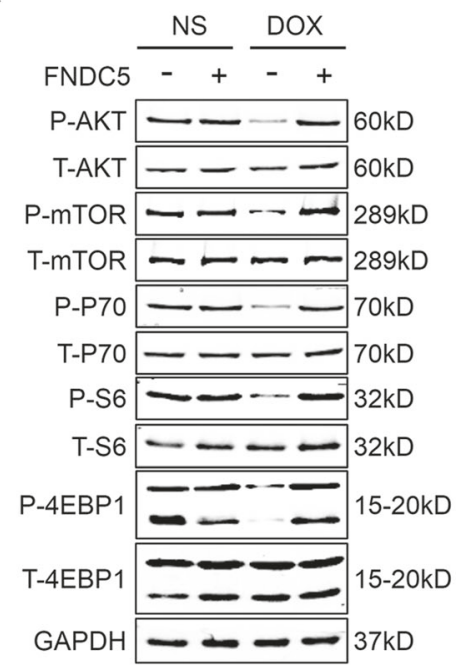

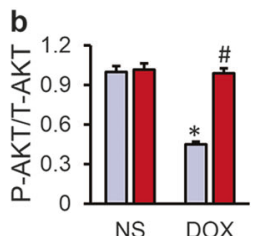

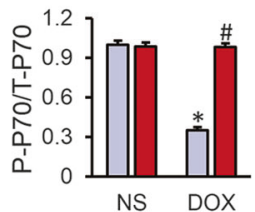

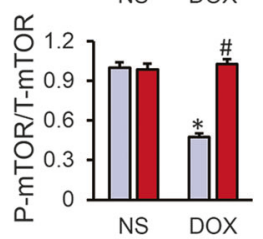

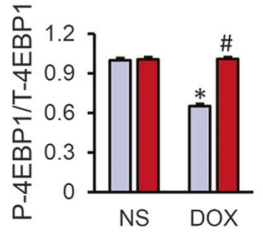

Fig. 4 FNDC5 activated AKT/mTOR signaling pathway in vivo and in vitro. a, b Western blots and quantitative results in murine hearts $(n=6)$. c, $\mathbf{d}$ Western blots and statistical results in cultured H9C 2 cells $(n=6)$. Values represent the mean \pm SEM. In figure $(\mathbf{a}, \mathbf{b}), * P<0.05$ versus the corresponding normal saline (NS) group mice injected with
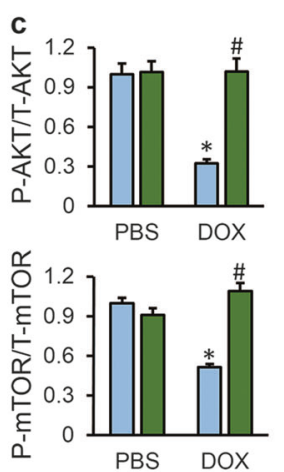

d
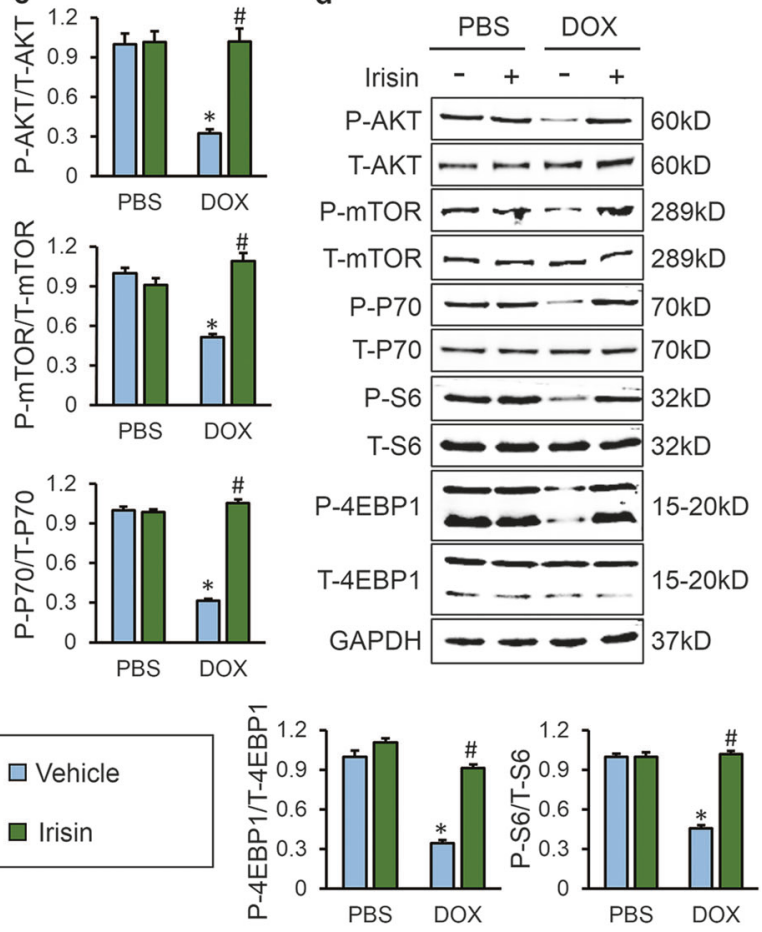

negative control (NC) adeno-associated virus $9,{ }^{\#} P<0.05$ versus DOX-treated mice injected with AAV9-NC. In figure (c, d), $* P<0.05$ versus PBS group treated with control vehicle, ${ }^{\#} P<0.05$ versus DOXtreated $\mathrm{H} 9 \mathrm{C} 2$ cells with irisin protection

abolished the beneficial role of FNDC5 on oxidative stress and cardiomyocyte apoptosis, whereas mTOR blockade by rapamycin only partly reversed the improvement of cardiomyocyte apoptosis by FNDC5 overexpression, with almost no effect on FNDC5-mediated anti-oxidant effects. In line with the data in vivo, we found that irisin lost both of the anti-oxidant and anti-apoptotic effects in cells treated with AKT inhibitor, whereas its anti-oxidant capacity was preserved in rapamycin-treated cells (Fig. S3a-f). Regarding the fact that mTOR is merely one of the downstream effector of AKT and mainly mediated the survival signals [14], we supposed that FNDC5 might exerted its antioxidant effect via a mechanism that is AKT-dependent, but mTOR-independent. Collectively, these data showed that AKT/mTOR signaling was responsible for FNDC5mediated protective role on cardiomyocyte apoptosis. DOX-treated hearts, and restoration of myocardial AKT blunted DOX-induced cardiac dysfunction [11]. Here, we observed that both myocardial overexpression of FNDC5 in vivo and irisin treatment in vitro mitigated DOX-induced inactivation of $\mathrm{AKT} / \mathrm{mTOR}$ pathway, further confirmed by the increased phosphorylation of P70/S6 and 4EBP1 (Fig. 4a-d). To gain evidence that the protective effects of FNDC5 were mediated by AKT/mTOR activation, we treated mice with either AKT inhibitor or rapamycin. As shown in Fig. 5a-g, AKT inhibition almost completely

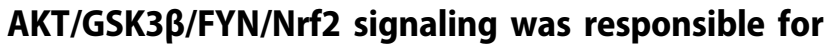 FNDC5-mediated protective role on oxidative damage}

Nrf2, a redox sensitive transcription factor, plays a pivotal role in redox homeostasis during oxidative stress [44]. Previous studies indicated that $N r f 2$ deficiency exacerbated DOX-induced cardiotoxicity, whereas $\mathrm{Nrf} 2$ activation 
a

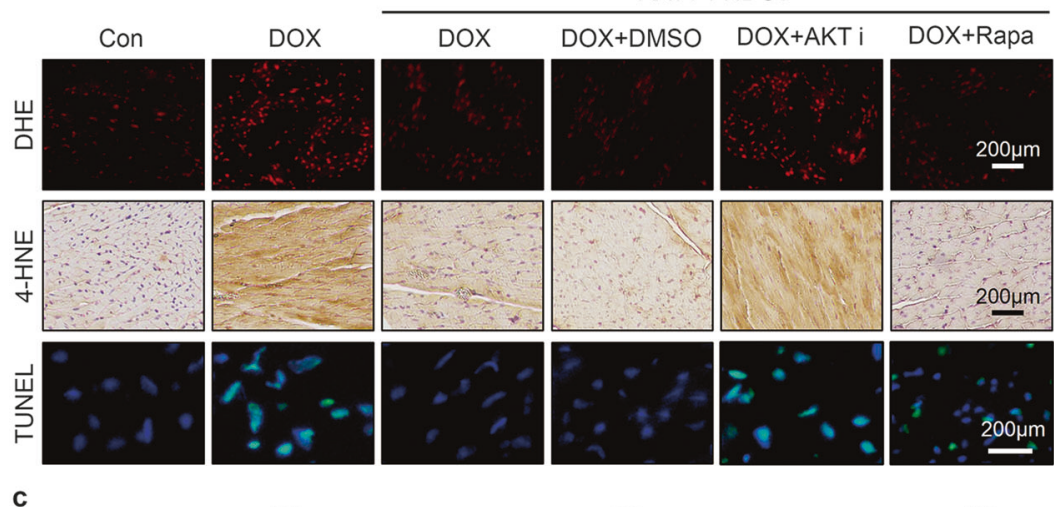

c
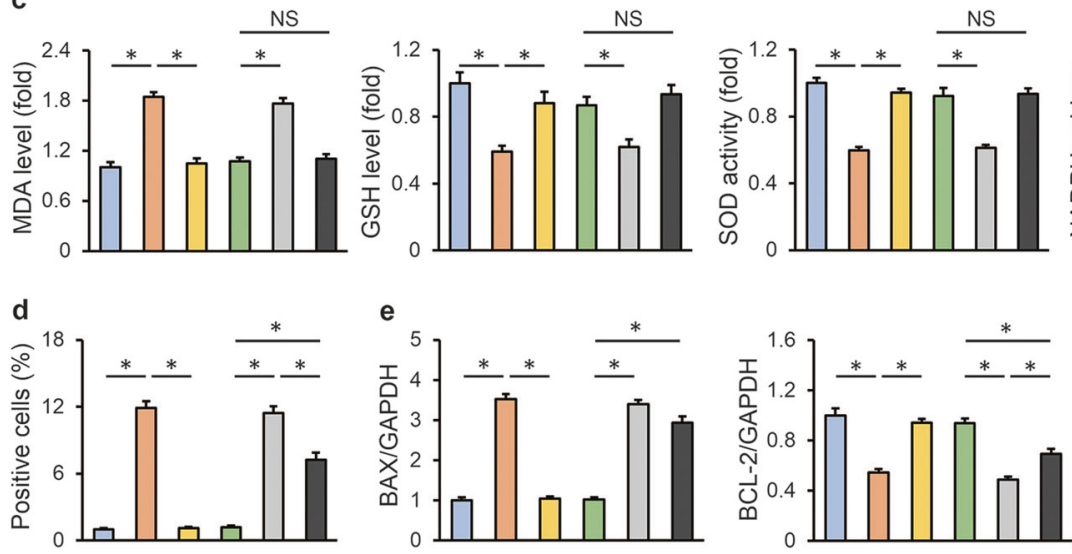

f

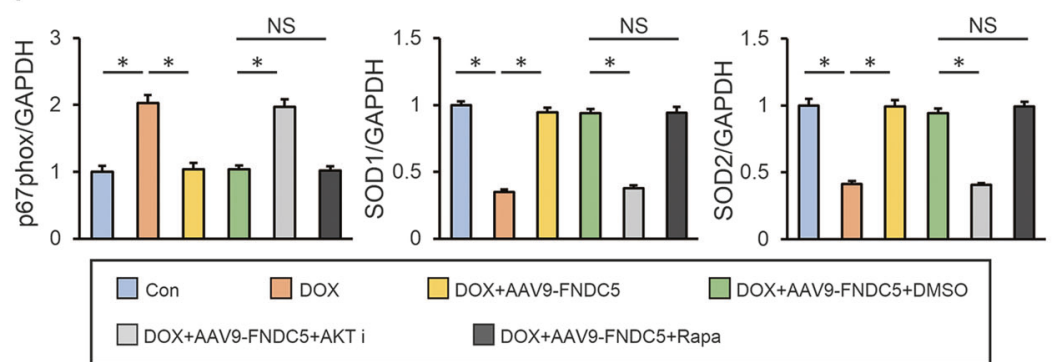

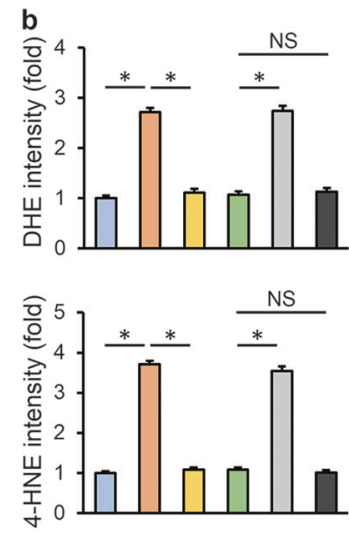
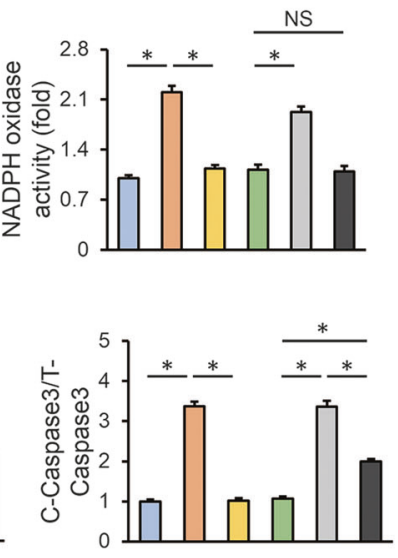

g

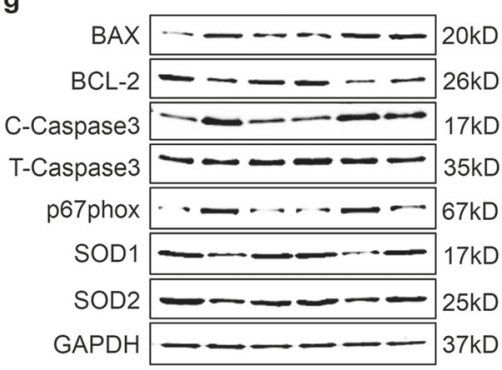

Fig. $5 \mathrm{AKT} / \mathrm{mTOR}$ signaling was responsible for FNDC5-mediated protective role on cardiomyocyte apoptosis. a Representative DHE, 4HNE, TUNEL staining images $(n=6)$. b Quantitative results of DHE and 4-HNE intensity $(n=6)$. c Quantitative results of myocardial

provided protection against cardiac dysfunction in response to DOX [7, 45]. Here, we found that irisin treatment blocked DOX-induced Nrf2 downregulation and preserved its transcriptional activity, as confirmed by the increased HO-1 protein level and Nqo1, Gclc, Gclm mRNA level (Fig. 6a-c). Keap1 is known to facilitate the ubiquitination and degradation of $\mathrm{Nrf} 2$ and regard as a major negative regulator of the antioxidant response [46]. However, we found that Keap1 expression was unaffected by irisin treatment (Fig. 6d). Consistent with the preserved transcriptional activity, we found that the protein level of Nrf2 in nucleus was increased with irisin incubation (Fig. 6a, e). As the mRNA level of Nrf2
MDA, GSH levels and SOD, NADPH oxidase activities $(n=6)$. d Quantitative results of TUNEL-positive cells $(n=6)$. e-g Western blots and statistical results $(n=6)$. Values represent the mean \pm SEM. $* P<0.05$ versus the matched group. NS means no significance

was also unaltered, we speculated that irisin might inhibit the nuclear export and degradation of Nrf2 (Fig. 6f). Numerous studies have defined a role of AKT/GSK3 $\beta / F Y N$ axis in the regulation of Nrf2 nuclear export [15-17]. And in view of the role of AKT in FNDC5-mediated anti-oxidant effect, we thus detected this axis in H9C2 cells. As shown in Fig. 6g-i, irisin treatment inactivated GSK3 $\beta$ and reduced FYN phosphorylation and its nuclear accumulation, which in turn decreased Nrf2 nuclear export and cytoplasmic degradation. In addition, $N r f 2$ deficiency also blocked the beneficial effect of irisin on oxidative stress, which is similar as AKT inhibitor (Fig. 6j, k). Consistent with the data in vitro, we also observed that 
a

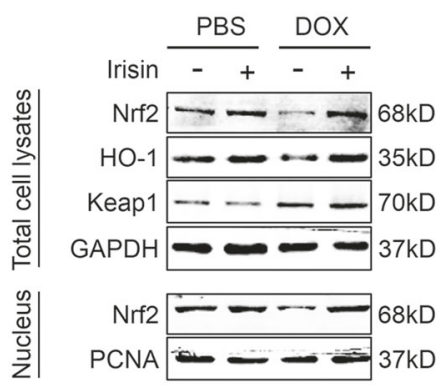

$9 \quad$ Irisin $\frac{\mathrm{PBS}}{-+} \frac{\mathrm{DOX}}{-+}$

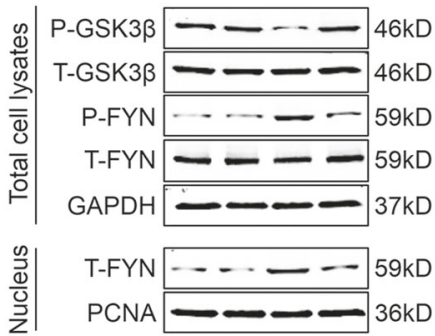

$\mathbf{k}$

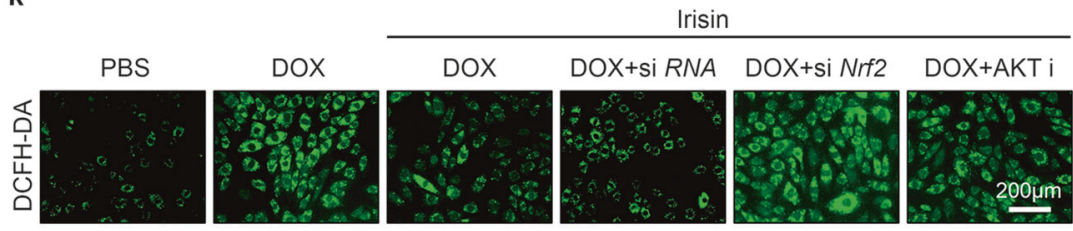

d
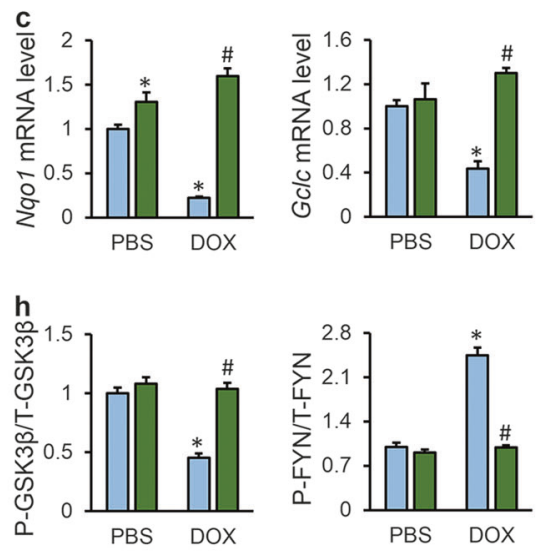
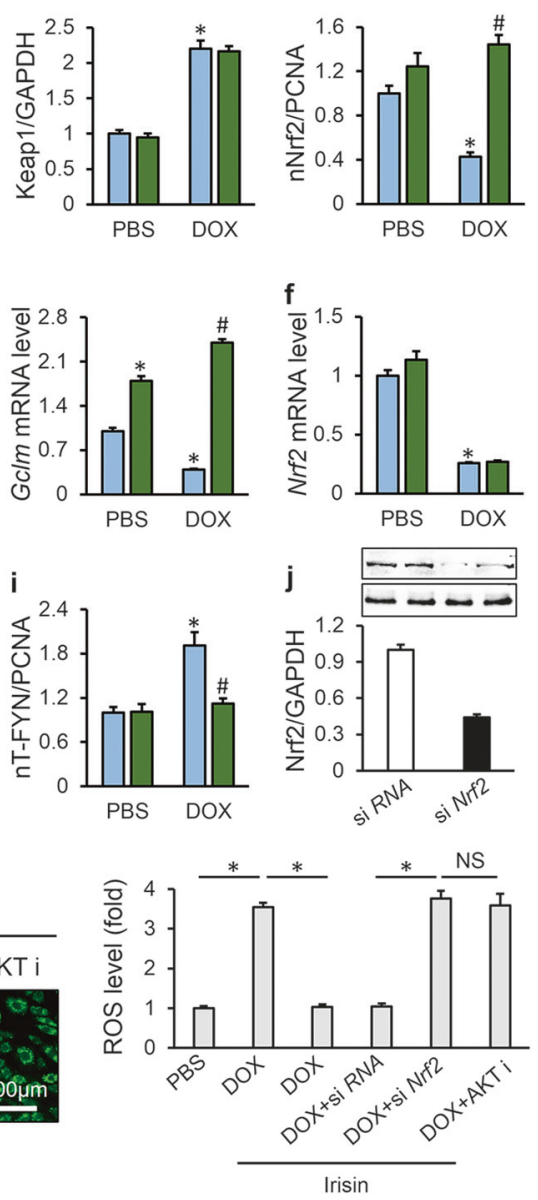

I

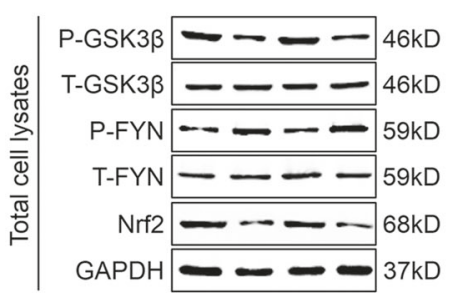

m

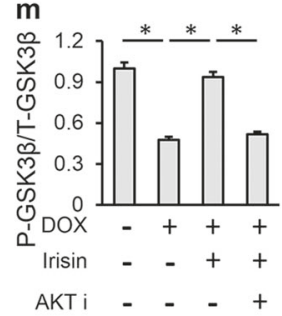

o

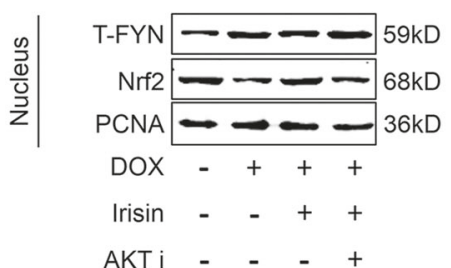

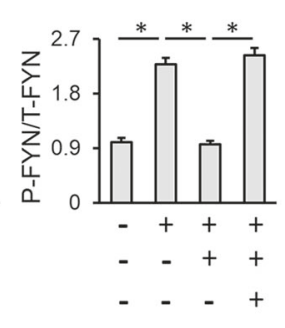

p

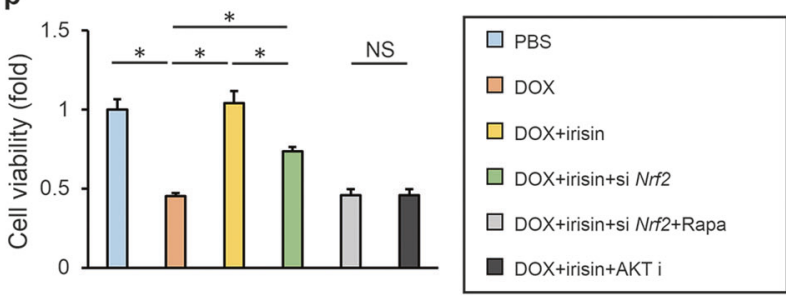

6). i FYN protein levels in nuclear lysates $(n=6)$. $\mathbf{j}$ Efficiency of si $N r f 2$ determined by western blots $(n=6)$. $\mathbf{k}$ DCFH-DA staining and the statistical data $(n=6)$. $1-0$ Western blots and quantitative results $(n=6)$. p Cell viability detected by CCK- 8 assay $(n=6)$. Values represent the mean \pm SEM. $* P<0.05$ versus the matched group. NS means no significance 
FNDC5 lost its anti-oxidant effect in Nrf2-deficient mice (Figure S4). To further verify the role of AKT in the regulation of $\mathrm{Nrf} 2$, we treated $\mathrm{H} 9 \mathrm{C} 2$ cells with an AKT inhibitor, and found that AKT inhibition resulted in GSK3 $\beta$ activation and promoted FYN nuclear translocation-mediated Nrf2 export and degradation, and abrogated the beneficial effect of irisin (Fig. 6k-o). Intriguingly, we found that Nrf2 deficiency partly abrogated irisin-mediated anti-apoptotic effect, nevertheless, Nrf2 deficiency together with mTOR inhibition exhibited a similar outcome on cardiomyocyte apoptosis as AKT inhibition (Fig. 6p). These data confirmed that AKT/ GSK3 $\beta / F Y N / N r f 2$ signaling was responsible for FNDC5mediated protective role on oxidative damage.

\section{HSP20 was involved in the activation of AKT caused by FNDC5}

Next, we investigated the possible mechanism by which FNDC5 activated AKT. We first detected PI3K activity, because it is suggested as an upstream activator of AKT and previous studies reported that FNDC5 activated AKT via PI3K [29, 47]. Unexpectedly, we found that irisin unaltered PI3K activity in the presence of DOX in H9C2 cells (Fig. 7a). HSPs play important roles in cellular stress resistance and exert protective effects on DOX-induced cardiac dysfunction [48]. Real-time PCR results implied that irisin significantly increased the mRNA level of $H s p 20$, with a slight increase of Hsp70 (Fig. 7b). Fan et al. previously demonstrated that HSP20 could interact with the phosphorylated AKT and prevent it from dephosphorylating by the phosphatase [12]. Herein, we observed that irisin also increased the protein level of HSP20 in response to DOX, whereas Hsp2O silence reduced irisin-mediated activation of $\mathrm{AKT}$, as confirmed by the two downstream effector, GSK3 $\beta$ and mTOR (Fig. 7c-f). Moreover, we also found that FNDC5 overexpressioninduced activation of AKT was abolished in Hsp20-deficient mice (Fig. S5a-b). Thus, we concluded that FNDC5 activated AKT via upregulation of HSP20.

\section{FNDC5/Irisin was a potential therapeutic agent against DOX-induced cardiotoxicity}

To enhance the clinical impact of our current work, we investigated whether infusion of exogenous irisin would attenuate DOX-induced cardiotoxicity. Administration of irisin markedly decreased DOX-triggered ROS overproduction and cardiomyocyte apoptosis (Fig. 8a-f). The decreased HW/TL and elevated serum cTnT level were both alleviated by irisin supplementation (Fig. 8g, h). Besides, the impaired heart function caused by DOX was also improved (Fig. 8i). More importantly, we found that systemic administration of irisin showed no hepatic toxicity in a

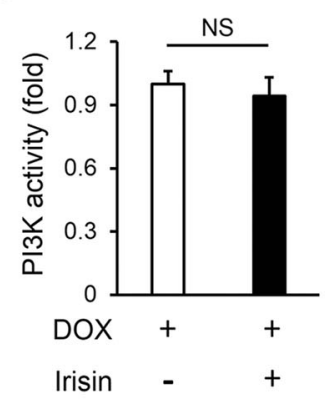

d

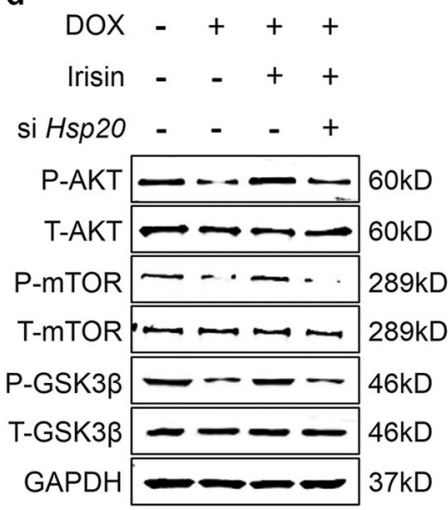

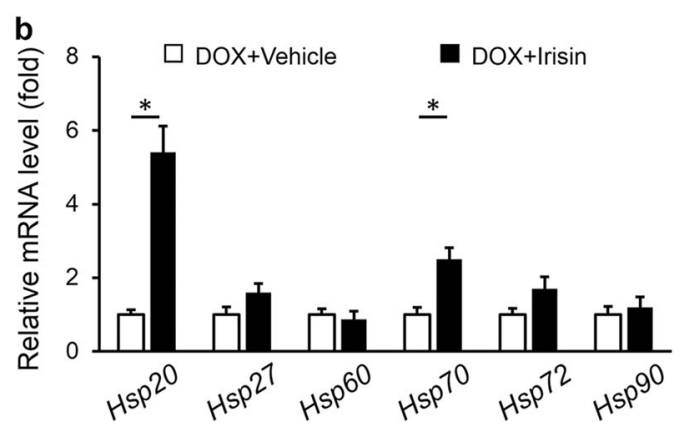

e
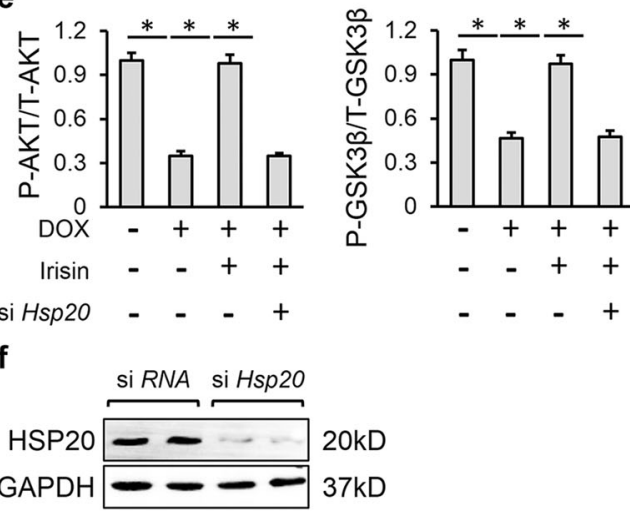

$20 \mathrm{kD}$

$37 \mathrm{kD}$

C
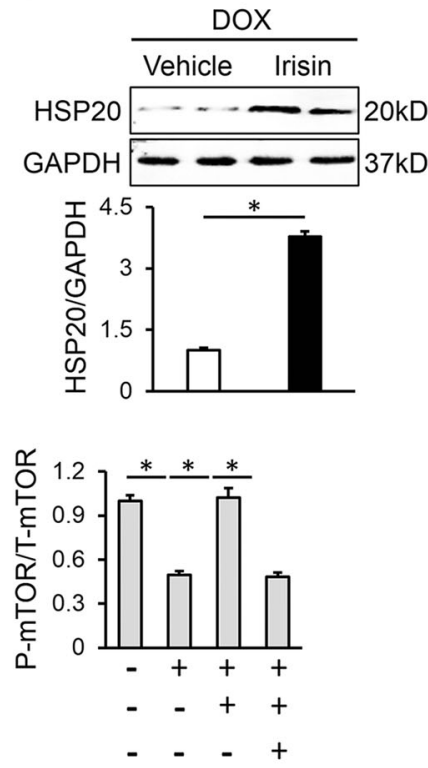

Fig. 7 HSP20 was involved in the activation of AKT caused by FNDC5. a Quantitative results of PI3K activity $(n=5)$. b Relative mRNA levels $(n=6)$. c HSP20 protein levels and the statistical results $(n=6)$. $\mathbf{d}, \mathbf{e}$ Western blots and quantitative data $(n=6)$. $\mathbf{f}$ Efficiency of si $H s p 20$ detected by western blots $(n=6)$. Values represent the mean \pm SEM. $* P<0.05$ versus the matched group. NS means no significance 
a

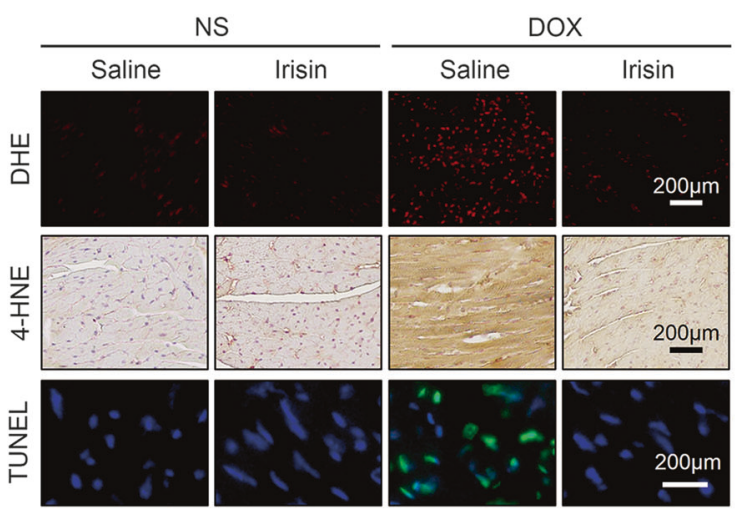

d

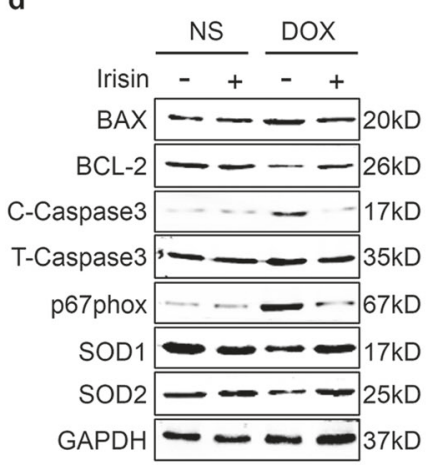

b
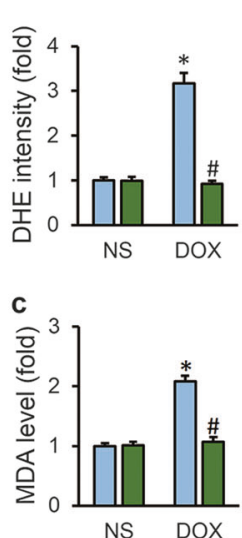

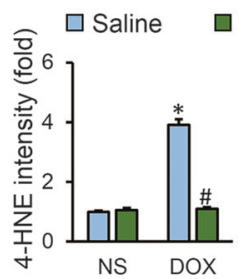

Irisin
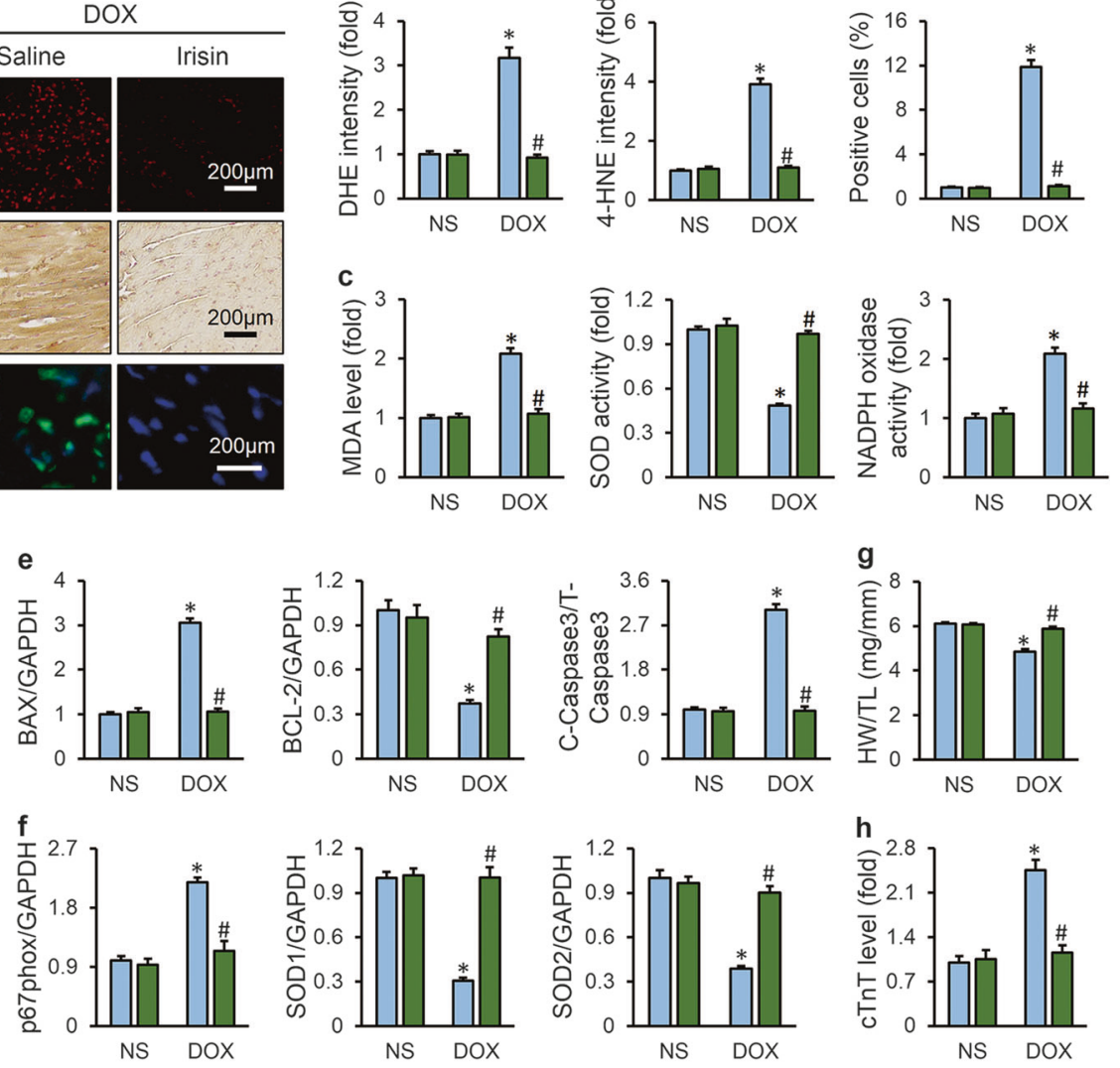
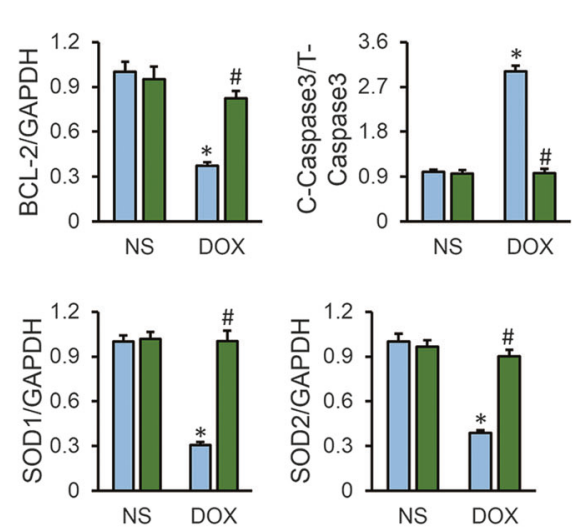

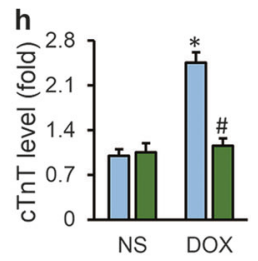

i
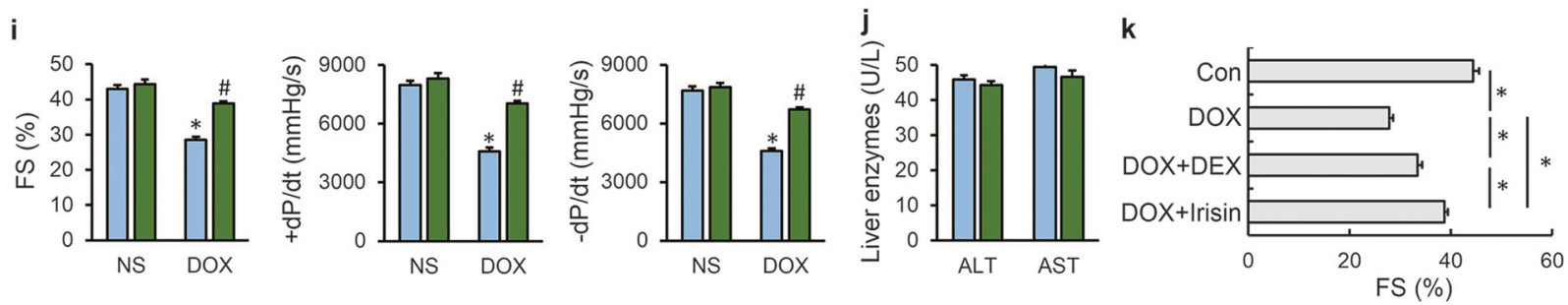

Fig. 8 FNDC5/Irisin was a potential therapeutic agent against DOXinduced cardiotoxicity. a, b Representative DHE, 4-HNE, TUNEL staining images and statistical results $(n=6)$. c Quantitative results of myocardial MDA levels and SOD, NADPH oxidase activities $(n=6)$. d-f Western blots and statistical results $(n=6)$. $\mathbf{g}$ Statistical results of the heart weight/tibia length $((\mathrm{HW} / \mathrm{TL})(n=8)$. (h) Serum cTnT levels

mice, as evaluated by the serum concentrations of liver enzymes (Fig. 8j). DEX is the only cardioprotective agent proven to be effective in the treatment of DOX-induced cardiotoxicity, we then compared the efficiency between irisin and DEX. As shown in Fig. 8k, irisin $(12 \mathrm{nmol} / \mathrm{kg} /$ day) worked better than DEX (60 mg/kg/week) in mice.

In addition to acute cardiac effects, von Hoff et al. observed that DOX therapy was also associated with chronic cardiotoxicity that occurred in about $1.7 \%$ of the patients [49]. We next assessed whether FNDC5 overexpression could alleviate cardiac dysfunction in a chronic model of DOX-induced cardiotoxicity. As shown in Fig. S6a-b, FNDC5 overexpression ameliorated cardiac $(n=6)$. i Echocardiographic and hemodynamic parameters $(n=8)$. j Serum liver enzymes levels $(n=8)$. k Fractional shortening (FS) of mice as determined via echocardiography $(n=8)$. Values represent the mean \pm SEM. $* P<0.05$ versus the corresponding normal saline (NS) group mice treated with saline, ${ }^{\#} P<0.05$ versus DOX-treated mice treated with irisin. In figure $(\mathbf{k}), * P<0.05$ versus the matched group

dysfunction and HW reduction in response to chronic DOX treatment. Moreover, we observed that FNDC5 overexpression also mitigated the mortality in response to repeated DOX injection (Fig. S6c). In line with the effect in acute model, we found that irisin was more effective than DEX in chronic cardiotoxicity (Fig. S6d). Considering the fact that long-term use of high-doses of DEX resulted in severe side effects, such as hepatic toxicity, occurrence of second malignancy and early death, we then investigated whether combined use of DEX with irisin could decrease the usage of DEX. As shown in Fig. S6e, combined use of DEX $(30 \mathrm{mg} / \mathrm{kg} /$ week $)$ with irisin $(6 \mathrm{nmol} / \mathrm{kg} /$ day $)$ could decrease the dosage of DEX, thereby might alleviate DEX- 
triggered side effects. All the data proved that FNDC5/Irisin was a potential therapeutic agent against DOX-induced cardiotoxicity.

\section{Discussion}

In the present study, we found that DOX treatment decreased myocardial level of FNDC5 and cardiac-specific overexpression of FNDC5 or irisin supplementation alleviated oxidative stress and cardiomyocyte apoptosis in DOX-induced cardiotoxicity in mice. Consistent with the data in vivo, irisin treatment exerted similar beneficial effects on DOX-induced oxidative damage and cardiomyocyte apoptosis in $\mathrm{H} 9 \mathrm{C} 2$ cells. Mechanistically, we identified that FNDC5/Irisin activated AKT/mTOR signaling and decreased DOX-induced cardiomyocyte apoptosis, and moreover, we provided direct evidence that the antioxidant effect of FNDC5/Irisin was mediated by the AKT/ GSK3 $\beta / F Y N / N r f 2$ axis in an mTOR-independent manner. And we also demonstrated that HSP20 was responsible for the activation of AKT caused by FNDC5/Irisin. Based on these findings, we supposed that FNDC5/Irisin was a potential therapeutic agent against DOX-induced cardiotoxicity.

FNDC5/Irisin is a recently identified exercise-induced myokine, which improves systemic metabolism via increasing energy expenditure and serves as an appealing therapeutic target for treatment of metabolic disorders $[18,20,30]$. However, emerging evidence identified an essential role of FNDC5/Irisin in the regulation of heart development and function. Farzaneh et al. found that the mRNA level of Fndc5 was increased during the process of cardiac differentiation, and overexpression of FNDC5 significantly increased whereas Fndc5 deficiency decreased cardiomyocyte differentiation rate of mouse embryonic stem cells [50-52]. In addition, several researches have proved that FNDC5/Irisin played a pivotal role in protecting the heart against ischemia/reperfusion injury and could be a potentially candidate marker for myocardial infarction [23, 53, 54]. Irisin supplementation could also attenuate collagen deposition and ventricular function impairment in diabetic hearts [55]. Recently, results from Li et al. suggested that irisin alleviated pressure overloadinduced cardiac hypertrophy in mice [24]. Previous studies indicated that DOX showed a specific affinity to the heart and the heart is a preferential target of DOX [56, 57]. In addition, DOX treatment destroyed myocardial redox homeostasis, and resulted in vacuolar degeneration and apoptosis of cardiomyocytes [4]. However, there is no available data about the role of FNDC5/Irisin in DOXinduced cardiotoxicity. In the current study, we observed that $F n d c 5$ deficiency at baseline elicited oxidative stress and apoptosis in $\mathrm{H} 9 \mathrm{C} 2$ cells, imitating the phenotype of DOX-induced cardiomyopathy in vitro. Cardiac-specific overexpression of FNDC5 or irisin supplementation alleviated oxidative stress and cardiomyocyte apoptosis in DOX-induced cardiotoxicity in vivo and in vitro. Skeletal muscle has previously been recognized as the main source of FNDC5/Irisin, however, in this study, we observed that the expression of FNDC5 was more abundant in the myocardium, which was markedly reduced in response to DOX. Numerous studies identified the heart as an endocrine organ and named the secretomes from the heart as 'cardiokines', which are increasingly recognized as essential regulators of cardiac physiology and pathology [58]. Previous studies also suggested the existence of irisin-specific receptor on the membrane of cardiomyocytes [47]. Hence, we dare to speculate that FNDC5/Irisin might be one of the 'cardiokines' and exert the cardioprotective effect in autocrine or paracrine manners, however, this hypothesis must be experimentally validated.

Previous studies revealed that AKT inactivation was responsible for DOX-induced cardiotoxicity, and enhanced AKT phosphorylation promoted cardiomyocyte survival and prevented DOX-induced cardiac dysfunction [11, 12]. The results from our study showed that FNDC5 overexpression in murine hearts could activate AKT/mTOR and exert protective effects on cardiomyocyte apoptosis, which is consistent with a previous study suggested that mTOR inhibition was the major contributor to DOX-induced cardiotoxicity [14]. Oxidative stress is regarded as another main contributor to DOX-induced cardiotoxicity. DOX treatment inhibits mitochondrial biosynthesis-related gene expression, disrupts of mitochondrial structure and function, and hampers normal mitochondrial metabolism and repairment, which eventually contributes to increased ROS production. Besides, upregulation of nitric oxide synthase, enhanced NADPH oxidase activity and formation of DOXiron complexes are also responsible for DOX-triggered ROS generation [3, 5, 8]. We observed that FNDC5/Irisin overtly decreased DOX-elicited oxidative stress damage, which could be reversed by AKT inhibition, but not rapamycin. Previous studies reported that AKT dephosphorylation resulted in GSK3 $3 / \mathrm{FYN}$ activation and FYN nuclear accumulation, which then promoted the nuclear exclusion of Nrf2 and its degradation in cytoplasm [15-17]. In this study, we found that FNDC5/Irisin alleviated oxidative damage via AKT/GSK3 $\beta / F Y N / N r f 2$ axis. Nrf2 is generally considered as the key transcription factor against oxidative stress by enhancing anti-oxidant gene expression, including HO-1. HO-1 or its metabolites-bilirubin was previously shown to inhibit the expression of NADPH oxidase subunits and NADPH oxidase activity in vivo and in vitro [59-61]. Furthermore, $\mathrm{Li}$ et al. previously demonstrated that HO-1 activation inhibited p67phox expression 
and prevented its membrane translocation [62]. Consistent with these data, we found that FNDC5 overexpression or irisin supplementation significantly inhibited NADPH oxidase activity and decreased p67phox subunit expression. Collectively, our results defined FNDC5/Irisin as an antioxidant to prevent cardiomyocytes from oxidative damage.

However, the pathway through which FNDC5/Irisin activated AKT remains unclear in this study. PI3K was previously reported to be involved in irisin-mediated activation of AKT, however, we found that irisin treatment unaffected the PI3K activity in response to DOX, but increased the expression of HSP20. Fan et al. suggested that HSP20 might block the action of phosphatases on AKT dephosphorylation, and HSP20 overexpression could effectively suppress oxidative stress as well as cardiomyocyte apoptosis [12]. HSPs, as molecular chaperones, have been extensively investigated on protection of DOXinduced cardiotoxicity. HSP27 has been regarded as the endogenous anti-oxidant and anti-apoptotic protein against DOX and attenuated DOX-induced cardiac dysfunction in mice [63]. Our present study found that HSP20 was responsible for the beneficial role of irisin, and Hsp20 deficiency abolished irisin-mediated activation of AKT. In addition, we compared the efficiency between irisin and DEX in the protection of DOX-induced cardiotoxicity, and it was worth noting that irisin worked better than DEX with no hepatic toxicity detected, and combined irisin with DEX treatment could decrease the dosage of DEX in mice. The data from this study provided evidence that it was possible using irisin as an adjuvant or alternative agent for the therapy of DOX-induced cardiotoxicity. This notion was further supported by the fact that irisin administration enhanced tumor sensitivity to DOX [64].

In conclusion, FNDC5/Irisin might be a potential therapeutic agent against DOX-induced cardiotoxicity.

Acknowledgements This work was supported by grants from National Natural Science Foundation of China (No: 81470516, 81500184, and 81700254), the Key Project of the National Natural Science Foundation (No. 81530012), the Fundamental Research Funds for the Central Universities (No. 2042017kf0085, 2042015kf0073) and Scientific Action Plans for the Prevention and Treatment of Major DiseasesCardiovascular Diseases (2016ZX-008-01).

\section{Compliance with ethical standards}

Conflict of interest The authors declare that they have no conflict of interest.

Publisher's note: Springer Nature remains neutral with regard to jurisdictional claims in published maps and institutional affiliations.

Open Access This article is licensed under a Creative Commons Attribution 4.0 International License, which permits use, sharing, adaptation, distribution and reproduction in any medium or format, as long as you give appropriate credit to the original author(s) and the source, provide a link to the Creative Commons license, and indicate if changes were made. The images or other third party material in this article are included in the article's Creative Commons license, unless indicated otherwise in a credit line to the material. If material is not included in the article's Creative Commons license and your intended use is not permitted by statutory regulation or exceeds the permitted use, you will need to obtain permission directly from the copyright holder. To view a copy of this license, visit http://creativecommons. org/licenses/by/4.0/.

\section{References}

1. Carvalho C, Santos RX, Cardoso S, Correia S, Oliveira PJ, Santos MS, et al. Doxorubicin: the good, the bad and the ugly effect. Curr Med Chem. 2009;16:3267-85.

2. Silber JH, Barber G. Doxorubicin-induced cardiotoxicity. N Engl J Med. 1995;333:1359-60.

3. Zhang S, Liu X, Bawa-Khalfe T, Lu LS, Lyu YL, Liu LF, et al. Identification of the molecular basis of doxorubicin-induced cardiotoxicity. Nat Med. 2012;18:1639-42.

4. Takemura G, Fujiwara H. Doxorubicin-induced cardiomyopathy from the cardiotoxic mechanisms to management. Prog Cardiovasc Dis. 2007;49:330-52.

5. Mukhopadhyay P, Rajesh M, Batkai S, Patel V, Kashiwaya Y, Liaudet L, et al. CB1 cannabinoid receptors promote oxidative stress and cell death in murine models of doxorubicin-induced cardiomyopathy and in human cardiomyocytes. Cardiovasc Res. 2010;85:773-84.

6. Circu ML, Aw TY. Reactive oxygen species, cellular redox systems, and apoptosis. Free Radic Biol Med. 2010;48:749-62.

7. Zhao L, Qi Y, Xu L, Tao X, Han X, Yin L, et al. MicroRNA-140$5 p$ aggravates doxorubicin-induced cardiotoxicity by promoting myocardial oxidative stress via targeting Nrf2 and Sirt2. Redox Biol. 2018;15:284-96.

8. Octavia Y, Tocchetti CG, Gabrielson KL, Janssens S, Crijns HJ, Moens AL. Doxorubicin-induced cardiomyopathy: from molecular mechanisms to therapeutic strategies. J Mol Cell Cardiol. 2012;52:1213-25.

9. Yuan YP, Ma ZG, Zhang X, Xu SC, Zeng XF, Yang Z, et al. CTRP3 protected against doxorubicin-induced cardiac dysfunction, inflammation and cell death via activation of Sirt1. J Mol Cell Cardiol. 2018;114:38-47.

10. Song G, Ouyang G, Bao S. The activation of Akt/PKB signaling pathway and cell survival. J Cell Mol Med. 2005;9:59-71.

11. Kitamura Y, Koide M, Akakabe Y, Matsuo K, Shimoda Y, Soma $\mathrm{Y}$, et al. Manipulation of cardiac phosphatidylinositol 3-kinase (PI3K)/Akt signaling by apoptosis regulator through modulating IAP expression (ARIA) regulates cardiomyocyte death during doxorubicin-induced cardiomyopathy. J Biol Chem. 2014;289:2788-800.

12. Fan GC, Zhou X, Wang X, Song G, Qian J, Nicolaou P, et al. Heat shock protein 20 interacting with phosphorylated Akt reduces doxorubicin-triggered oxidative stress and cardiotoxicity. Circ Res. 2008;103:1270-9.

13. Shigemitsu K, Tsujishita Y, Hara K, Nanahoshi M, Avruch J, Yonezawa K. Regulation of translational effectors by amino acid and mammalian target of rapamycin signaling pathways. PossibInvolv autophagy Cult hepatoma cells J Biol Chem. 1999;274:1058-65.

14. Zhu W, Soonpaa MH, Chen H, Shen W, Payne RM, Liechty EA, et al. Acute doxorubicin cardiotoxicity is associated with p53induced inhibition of the mammalian target of rapamycin pathway. Circulation. 2009;119:99-106.

15. Dai X, Yan X, Zeng J, Chen J, Wang Y, Chen J, et al. Elevating CXCR7 improves angiogenic function of EPCs via Akt/GSK- 
3beta/Fyn-mediated Nrf2 activation in diabetic limb ischemia. Circ Res. 2017;120:e7-23.

16. Xin Y, Bai Y, Jiang X, Zhou S, Wang Y, Wintergerst KA, et al. Sulforaphane prevents angiotensin II-induced cardiomyopathy by activation of Nrf2 via stimulating the Akt/GSK-3ss/Fyn pathway. Redox Biol. 2018;15:405-17.

17. Jain AK, Jaiswal AK. GSK-3beta acts upstream of Fyn kinase in regulation of nuclear export and degradation of NF-E2 related factor 2. J Biol Chem. 2007;282:16502-10.

18. Bostrom P, Wu J, Jedrychowski MP, Korde A, Ye L, Lo JC, et al. A PGC1-alpha-dependent myokine that drives brown-fat-like development of white fat and thermogenesis. Nature. 2012;481:463-8.

19. Perakakis N, Triantafyllou GA, Fernandez-Real JM, Huh JY, Park $\mathrm{KH}$, Seufert J, et al. Physiology and role of irisin in glucose homeostasis. Nat Rev Endocrinol. 2017;13:324-37.

20. Liu TY, Xiong XQ, Ren XS, Zhao MX, Shi CX, Wang JJ, et al. FNDC5 alleviates hepatosteatosis by restoring AMPK/mTORmediated autophagy, fatty acid oxidation, and lipogenesis in mice. Diabetes. 2016;65:3262-75.

21. Zhang Y, Song H, Zhang Y, Wu F, Mu Q, Jiang M, et al. Irisin inhibits atherosclerosis by promoting endothelial proliferation through microRNA126-5p. J Am Heart Assoc. 2016;5:e004031.

22. Fu J, Han Y, Wang J, Liu Y, Zheng S, Zhou L, et al. Irisin lowers blood pressure by improvement of endothelial dysfunction via AMPK-Akt-eNOS-NO pathway in the spontaneously hypertensive rat. J Am Heart Assoc. 2016;5:e003433.

23. Wang H, Zhao YT, Zhang S, Dubielecka PM, Du J, Yano N, et al. Irisin plays a pivotal role to protect the heart against ischemia and reperfusion injury. J Cell Physiol. 2017;232:3775-85.

24. Li RL, Wu SS, Wu Y, Wang XX, Chen HY, Xin JJ, et al. Irisin alleviates pressure overload-induced cardiac hypertrophy by inducing protective autophagy via mTOR-independent activation of the AMPK-ULK1 pathway. J Mol Cell Cardiol. 2018;121:242-55.

25. Ling L, Chen D, Tong Y, Zang YH, Ren XS, Zhou H, et al. Fibronectin type III domain containing 5 attenuates NLRP3 inflammasome activation and phenotypic transformation of adventitial fibroblasts in spontaneously hypertensive rats. J Hypertens. 2018;36:1104-14.

26. Chen K, Xu Z, Liu Y, Wang Z, Li Y, Xu X, et al. Irisin protects mitochondria function during pulmonary ischemia/reperfusion injury. Sci Transl Med. 2017;9:eaao6298.

27. He Y, Peng X, Wu T, Yang W, Liu W, Zhang J, et al. Restricting the induction of NGF in ovarian stroma engenders selective follicular activation through the mTOR signaling pathway. Cell Death Dis. 2017;8:e2817.

28. Volkers M, Konstandin MH, Doroudgar S, Toko H, Quijada P, Din S, et al. Mechanistic target of rapamycin complex 2 protects the heart from ischemic damage. Circulation. 2013;128:2132-44.

29. Liu TY, Shi CX, Gao R, Sun HJ, Xiong XQ, Ding L, et al. Irisin inhibits hepatic gluconeogenesis and increases glycogen synthesis via the PI3K/Akt pathway in type 2 diabetic mice and hepatocytes. Clin Sci (Lond). 2015;129:839-50.

30. Tang H, Yu R, Liu S, Huwatibieke B, Li Z, Zhang W. Irisin inhibits hepatic cholesterol synthesis via AMPK-SREBP2 signaling. Ebiomedicine. 2016;6:139-48.

31. Lue Y, Gao C, Swerdloff R, Hoang J, Avetisyan R, Jia Y, et al. Humanin analog enhances the protective effect of dexrazoxane against doxorubicin-induced cardiotoxicity. Am J Physiol Heart Circ Physiol. 2018;315:H634-43.

32. Fisher PW, Salloum F, Das A, Hyder H, Kukreja RC. Phosphodiesterase-5 inhibition with sildenafil attenuates cardiomyocyte apoptosis and left ventricular dysfunction in a chronic model of doxorubicin cardiotoxicity. Circulation. 2005;111:1601-10.
33. Zhang X, Ma ZG, Yuan YP, Xu SC, Wei WY, Song P, et al. Rosmarinic acid attenuates cardiac fibrosis following long-term pressure overload via AMPKalpha/Smad3 signaling. Cell Death Dis. 2018;9:102.

34. Ma ZG, Zhang X, Yuan YP, Jin YG, Li N, Kong CY, et al. A77 1726 (leflunomide) blocks and reverses cardiac hypertrophy and fibrosis in mice. Clin Sci (Lond). 2018;132:685-99.

35. Ma ZG, Yuan YP, Zhang X, Xu SC, Wang SS, Tang QZ. Piperine attenuates pathological cardiac fibrosis via PPAR-gamma/AKT pathways. Ebiomedicine. 2017;18:179-87.

36. Ma ZG, Dai J, Wei WY, Zhang WB, Xu SC, Liao HH, et al. Asiatic acid protects against cardiac hypertrophy through activating AMPKalpha signalling pathway. Int $\mathrm{J}$ Biol Sci. 2016;12:861-71.

37. Ma ZG, Dai J, Zhang WB, Yuan Y, Liao HH, Zhang N, et al. Protection against cardiac hypertrophy by geniposide involves the GLP-1 receptor/AMPKalpha signalling pathway. Br J Pharmacol. 2016;173:1502-16.

38. Ma ZG, Yuan YP, Xu SC, Wei WY, Xu CR, Zhang X, et al. CTRP3 attenuates cardiac dysfunction, inflammation, oxidative stress and cell death in diabetic cardiomyopathy in rats. Diabetologia. 2017;60:1126-37.

39. Ma ZG, Dai J, Yuan YP, Bian ZY, Xu SC, Jin YG, et al. T-bet deficiency attenuates cardiac remodelling in rats. Basic Res Cardiol. 2018;113:19.

40. Fan D, Yang Z, Liu FY, Jin YG, Zhang N, Ni J, et al. Sesamin protects against cardiac remodeling via Sirt3/ROS pathway. Cell Physiol Biochem. 2017;44:2212-27.

41. Wang Z, Wang M, Liu J, Ye J, Jiang H, Xu Y, et al. Inhibition of TRPA1 attenuates doxorubicin-induced acute cardiotoxicity by suppressing oxidative stress, the inflammatory response, and endoplasmic reticulum stress. Oxid Med Cell Longev. 2018;2018:5179468

42. Simons JP, Schols AM, Buurman WA, Wouters EF. Weight loss and low body cell mass in males with lung cancer: relationship with systemic inflammation, acute-phase response, resting energy expenditure, and catabolic and anabolic hormones. Clin Sci (Lond). 1999;97:215-23.

43. Kratz F, Roth T, Fichiner I, Schumacher P, Fiebig HH, Unger C. In vitro and in vivo efficacy of acid-sensitive transferrin and albumin doxorubicin conjugates in a human xenograft panel and in the MDA-MB-435 mamma carcinoma model. J Drug Target. 2000;8:305-18.

44. Ma Q. Role of nrf2 in oxidative stress and toxicity. Annu Rev Pharm Toxicol. 2013;53:401-26.

45. Li S, Wang W, Niu T, Wang H, Li B, Shao L, et al. Nrf2 deficiency exaggerates doxorubicin-induced cardiotoxicity and cardiac dysfunction. Oxid Med Cell Longev. 2014;2014:748524.

46. McMahon M, Itoh K, Yamamoto M, Hayes JD. Keap1-dependent proteasomal degradation of transcription factor Nrf2 contributes to the negative regulation of antioxidant response element-driven gene expression. J Biol Chem. 2003;278:21592-600.

47. Xie C, Zhang Y, Tran TD, Wang H, Li S, George EV, et al. Irisin controls growth, intracellular $\mathrm{Ca} 2+$ signals, and mitochondrial thermogenesis in cardiomyoblasts. Plos One. 2015;10:e136816.

48. Liu L, Zhang X, Qian B, Min X, Gao X, Li C, et al. Overexpression of heat shock protein 27 attenuates doxorubicininduced cardiac dysfunction in mice. Eur $\mathrm{J}$ Heart Fail. 2007;9:762-9.

49. Von Hoff DD, Layard MW, Basa P, Davis HJ, Von Hoff AL, Rozencweig M, et al. Risk factors for doxorubicin-induced congestive heart failure. Ann Intern Med. 1979;91:710-7.

50. Zadegan FG, Ghaedi K, Kalantar SM, Peymani M, Hashemi MS, Baharvand $\mathrm{H}$, et al. Cardiac differentiation of mouse embryonic stem cells is influenced by a PPAR gamma/PGC-1alpha-FNDC5 
pathway during the stage of cardiac precursor cell formation. Eur J Cell Biol. 2015;94:257-66.

51. Rabiee F, Forouzanfar M, Ghazvini ZF, Tanhaei S, Ghaedi K, Motovali BM, et al. Induced expression of Fndc5 significantly increased cardiomyocyte differentiation rate of mouse embryonic stem cells. Gene. 2014;551:127-37.

52. Nazem S, Rabiee F, Ghaedi K, Babashah S, Sadeghizadeh M, Nasr-Esfahani MH. Fndc5 knockdown induced suppression of mitochondrial integrity and significantly decreased cardiac differentiation of mouse embryonic stem cells. J Cell Biochem. 2018;119:4528-39.

53. Wang Z, Chen K, Han Y, Zhu H, Zhou X, Tan T, et al. Irisin protects heart against ischemia-reperfusion injury through a SOD2-dependent mitochondria mechanism. J Cardiovasc Pharmacol. 2018;72:259-69.

54. Kuloglu T, Aydin S, Eren MN, Yilmaz M, Sahin I, Kalayci M, et al. Irisin: a potentially candidate marker for myocardial infarction. Peptides. 2014;55:85-91.

55. Liu X, Mujahid H, Rong B, Lu QH, Zhang W, Li P, et al. Irisin inhibits high glucose-induced endothelial-to-mesenchymal transition and exerts a dose-dependent bidirectional effect on diabetic cardiomyopathy. J Cell Mol Med. 2018;22:808-22.

56. Lewis W, Galizi M, Puszkin S. Compartmentalization of adriamycin and daunomycin in cultured chick cardiac myocytes. Eff Synth Contract cytoplasmic proteins Circ Res. 1983;53:352-62.

57. Stewart DJ, Grewaal D, Green RM, Mikhael N, Goel R, Montpetit VA, et al. Concentrations of doxorubicin and its metabolites in human autopsy heart and other tissues. Anticancer Res. 1993;13:1945-52.

58. Yan W, Guo Y, Tao L, Lau WB, Gan L, Yan Z, et al. C1q/tumor necrosis factor-related protein-9 regulates the fate of implanted mesenchymal stem cells and mobilizes their protective effects against ischemic heart injury via multiple novel signaling pathways. Circulation. 2017;136:2162-77.

59. Datla SR, Dusting GJ, Mori TA, Taylor CJ, Croft KD, Jiang F. Induction of heme oxygenase-1 in vivo suppresses NADPH oxidase derived oxidative stress. Hypertension. 2007;50: 636-42.

60. Lu CY, Yang YC, Li CC, Liu KL, Lii CK, Chen HW. Andrographolide inhibits TNFalpha-induced ICAM-1 expression via suppression of NADPH oxidase activation and induction of HO-1 and GCLM expression through the PI3K/Akt/Nrf2 and PI3K/Akt/ AP-1 pathways in human endothelial cells. Biochem Pharmacol. 2014;91:40-50.

61. Fujii M, Inoguchi T, Sasaki S, Maeda Y, Zheng J, Kobayashi K, et al. Bilirubin and biliverdin protect rodents against diabetic nephropathy by downregulating $\mathrm{NAD}(\mathrm{P}) \mathrm{H}$ oxidase. Kidney Int. 2010;78:905-19.

62. Li X, Schwacha MG, Chaudry IH, Choudhry MA. Heme oxygenase-1 protects against neutrophil-mediated intestinal damage by down-regulation of neutrophil p47phox and p67phox activity and $\mathrm{O} 2$ - production in a two-hit model of alcohol intoxication and burn injury. J Immunol. 2008;180:6933-40.

63. Turakhia S, Venkatakrishnan CD, Dunsmore K, Wong H, Kuppusamy P, Zweier JL, et al. Doxorubicin-induced cardiotoxicity: direct correlation of cardiac fibroblast and $\mathrm{H} 9 \mathrm{c} 2$ cell survival and aconitase activity with heat shock protein 27 . Am J Physiol Heart Circ Physiol. 2007;293:H3111-21.

64. Gannon NP, Vaughan RA, Garcia-Smith R, Bisoffi M, Trujillo KA. Effects of the exercise-inducible myokine irisin on malignant and non-malignant breast epithelial cell behavior in vitro. Int $\mathbf{J}$ Cancer. 2015;136:E197-202. 